\title{
Major Vegetation Types of the Soutpansberg Conservancy and the Blouberg Nature Reserve, South Africa
}

\author{
THEO H.C. MOSTERT \\ GEORGE J. BREDENKAMP \\ HANNES L. KLOPPER \\ CORNIE VERWEY \\ ${ }^{1}$ African Vegetation and Plant Diversity Research Centre \\ Department of Botany \\ University of Pretoria \\ South Africa \\ RACHEL E. MOSTERT \\ Directorate Nature Conservation \\ Gauteng Department of Agriculture \\ Conservation and Environment \\ South Africa \\ NORBERT HAHN ${ }^{1}$ \\ Correspondence to: Theo Mostert \\ e-mail: tmostert@tuks.co.za
}

Postal Address: African Vegetation and Plant Diversity Research Centre, Department of Botany, University of Pretoria, Pretoria, 0002

\begin{abstract}
The Major Megetation Types (MVT) and plant communities of the Soutpansberg Centre of Endemism are described in detail, with special reference to the Soutpansberg Conservancy and the Blouberg Nature Reserve. Phytosociological data from 442 sample plots were ordinated using a DEtrended CORrespondence ANAlysis (DECORANA) and classified using TWo-Way INdicator SPecies ANalysis (TWINSPAN). The resulting classification was further refined with table-sorting procedures based on the Braun-Blanquet floristic-sociological approach of vegetation classification using MEGATAB. Eight MVT's were identified and described as Eragrostis lehmanniana var. lehmanniana-Sclerocarya birrea subsp. caffra Blouberg Northern Plains Bushveld, Euclea divinorum-Acacia tortilis Blouberg Southern Plains Bushveld, Englerophytum magalismontanum-Combretum molle Blouberg Mountain Bushveld, Adansonia digitata-Acacia nigrescens Soutpansberg Arid Northern Bushveld, Catha edulis-Flueggia virosa Soutpansberg Moist Mountain Thickets, Diplorhynchus condylocarpon-Burkea africana Soutpansberg Leached Sandveld, Rhus rigida var. rigida-Rhus magalismontanum subsp. coddii Soutpansberg Mistbelt Vegetation and Xymalos monospora-Rhus chirendensis Soutpansberg Forest Vegetation.
\end{abstract}

Keywords: Phytosociology, vegetation ecology, grassland, savanna, thicket

The Soutpansberg Conservancy (SC) and the Blouberg Nature Reserve (BNR) reveal extremely rich diversities of plant communities relative to the sizes of these conservation areas (Van Wyk \& Smith 2001). Although Van Rooyen and Bredenkamp (1996) recognised this diversity and unique composition of plant communities within the Soutpansberg-Blouberg complex, the lack of detailed research in the region forced them to merge the area's vegetation under the broad term of Soutpansberg Arid Mountain Bushveld. Acocks (1953) recognised four different Veld Types for the greater surrounding region and described them as Arid Sweet Bushveld, Mixed Bushveld, Sourish Mixed Bushveld and Sour Bushveld. Most of these Veld Types were described as heterogeneous (Acocks 1953), comprising many sub-communities with different agricultural and production potentials. In addition to the savanna vegetation of the area, Geldenhuys and Murray (1993) and Lubke and McKenzie (1996) described and mapped the patches of Afromontane Forest associated with the region. Van Wyk and Smith (2001) only briefly mention the occurrence of "Fynbos-type" vegetation along the summit of the mountain. They also refer to dense, almost monospecific stands of Lebombo ironwood (Androstachys johnsonii) on the arid northern slopes of the mountain. Due to major gaps in the available vegetation data, no attempt has yet been made to synthesise, classify and to describe the plant communities of this region.

\section{THE STUDY AREA}

Location

The BNR (7 $000 \mathrm{ha})$ and SC (100 $000 \mathrm{ha})$ are located in the arid northern regions of the Limpopo Province of South Africa (Fig. 1). For the purpose of this study the SC is defined as a section of the Soutpansberg Mountain Range contained by four provincial and national roads. The eastern boundary (E29 $\left.55^{\prime}\right)$ is the N1 from Makhado in the south to Wyllies Poort in the north. The northern boundary (S22 $\left.{ }^{\circ} 2^{\prime}\right)$ is the R523 road from Wyllies Poort in the east to Kalkheuwel in the west. The western boundary $\left(\mathrm{E} 29^{\circ} 15^{\prime}\right)$ is the R521 road from Kalkheuwel in the north to Vivo in the south. The southern boundary $\left(\mathrm{S}_{2} 3^{\circ} 12^{\prime}\right)$ is the R522 road from Vivo in the west to Makhado in the east. From east to west it spans approximately $70 \mathrm{~km}$ and from north to south approximately $25 \mathrm{~km}$ at its widest. The BNR lies approximately $40 \mathrm{~km}$ to the west, situated along the eastern half of the Blouberg Mountain.

\section{Topography}

The SC and BNR are part of the Blouberg-Soutpansberg Mountain Range, with ENE-WSW orientation. Although the Blouberg and Soutpansberg belong to the same geological formation they are referred to as separate entities. Successive faulting along the Tshamuvhudzi, Kranspoort, Nakab and Zoutpan strike-faults, followed by the northwards tilting of the 


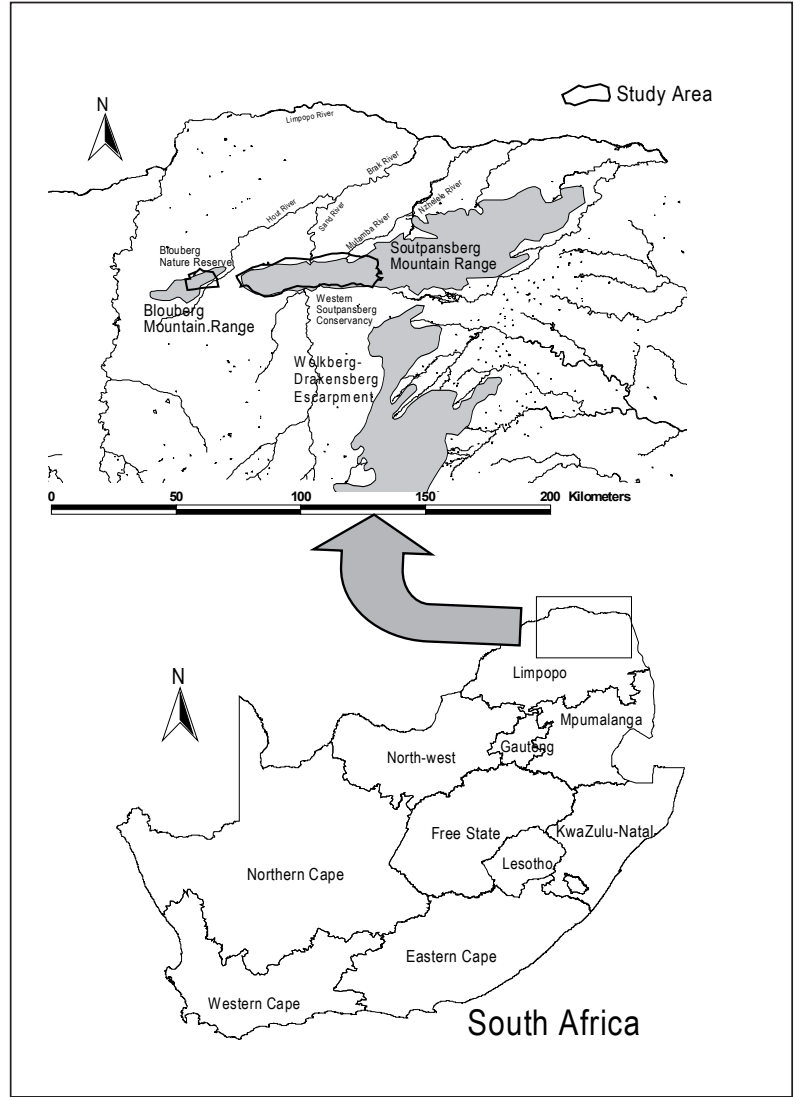

FIGURE 1

The location of the Blouberg Nature Reserve and Western Soutpansberg Conservancy within South Africa.

area, created these quartzite mountains within the surrounding Limpopo Plain. This gave the Blouberg-Soutpansberg Mountain Range a wedge-shaped appearance with steep southern slopes and moderate northern slopes. The ridges are highest at the western extremity of this range, which gradually descend until they finally plunge beneath the Karoo Supergroup along the northern reaches of the Lebombo Mountains near the northeastern border of the Limpopo Province. The SC's altitude ranges from $750 \mathrm{~m}$ above sea level at Waterpoort to $1748 \mathrm{~m}$ at Lejuma. The BNR's altitude ranges from $850 \mathrm{~m}$ above sea level in the east to $1400 \mathrm{~m}$ in the west. The highest peak of the Blouberg lies further to the west, and reaches $2051 \mathrm{~m}$ above sea level (Bumby 2000). The surrounding plains are approximately $850 \mathrm{~m}$ above sea level.

\section{Geology and soils}

The geology of the SC and BNR is dominated by pink, erosionresistant quartzite, and sandstone, with minor pebble washes of the Wyllies Poort Geological Formation of the Soutpansberg Group. Other less prominent rock types include shale, conglomerate, basalt and diabase intrusions. The rocks of the study area do not contain large amounts of minerals that are of economic value.

Soils derived from quartzite and sandstone are generally shallow, gravelly, skeletal and well drained, with low nutrient content and acidic characteristics. Soils derived from the basalt and diabase dykes are fine-textured, clayey, well weathered, and generally deep. These poorly drained soils are prone to erosion along the higher rainfall southern slopes. Soils derived from the Aeolian Kalahari sands are fine-grained deep sands. Large areas along the northern slope contain no soil, and comprise only the exposed underlying mother material. Peat soils occur along the cooler high lying wetlands of the SC. The deeper soils within the mistbelt act as sponge areas, which slowly release water to feed mountain streams over extended periods.

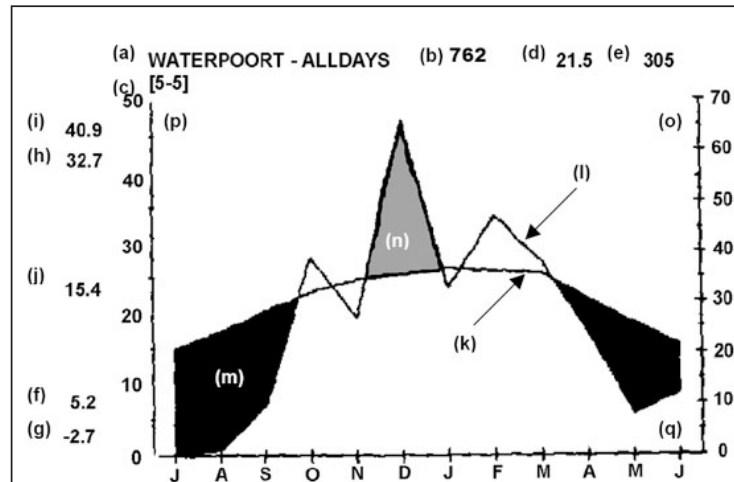

Legend

\begin{tabular}{|l|l|}
\hline a Weather station name & j Average daily temp fluctuation \\
\hline b Altitude & k Average monthly temp \\
\hline $\begin{array}{l}\text { c Number of years recorded } \\
\text { [temp - rainfall] }\end{array}$ & I Average monthly precipitation \\
\hline d Average annual temp $\left({ }^{\circ} \mathrm{C}\right)$ & m Dry season \\
\hline e Average annual rainfall $(\mathrm{mm})$ & $\mathrm{n}$ Wet season \\
\hline $\begin{array}{l}\text { f Average daily min (coldest } \\
\text { month) }\end{array}$ & o Rainfall axis \\
\hline g Lowest temp recorded & $\mathrm{p}$ Temperature axis \\
\hline $\begin{array}{l}\text { h Average daily max (warmest } \\
\text { month) }\end{array}$ & q Time axis (months) \\
\hline i Highest temp recorded & \\
\hline
\end{tabular}

FIGURE 2

Climate diagram for the Alldays/Waterpoort area

\section{Climate}

The SC and BNR fall within the summer rainfall zone of southern Africa. A climate diagram (Fig. 2) depicts typical rainfall patterns and temperature gradients within the study area. Due to the east-west orientation of the Soutpansberg it experiences orographic rainfall. This phenomenon is due to moisture-laden air from the Indian Ocean, driven by the prevailing south-easterly winds into the southern scarp of the Soutpansberg. The north-south orientated WolkbergDrakensberg escarpment further blocks the westerly movement of the atmospheric moisture, forcing it into the wedge created by the two mountain systems in the vicinity of Entabeni. Large amounts of rain are discharged onto these southern slopes of the Soutpansberg and eastern slopes of the WolkbergDrakensberg escarpment. Entabeni receives an annual rainfall of $1874 \mathrm{~mm}$. Orographic mist along this southern slope may increase annual precipitation to $3233 \mathrm{~mm}$ (Hahn 2002; Olivier \& Rautenbach 2002). This creates a rain-shadow effect along the western slopes of the Wolkberg-Drakensberg escarpment and the northern slopes of the Soutpansberg. With the SC and BNR located northwest of the Soutpansberg-WolkbergDrakensberg escarpment junction, a double rain-shadow effect is experienced along the northern slopes of the study area. Waterpoort, located north of the Soutpansberg, receives only $367 \mathrm{~mm}$ rain annually.

Due to the extreme topographic diversity and altitude changes over short distances within the study area, the climate (especially rainfall and mist precipitation) varies dramatically. The amount of orographic rain associated with the southern ridges varies considerably in accordance to the changing landscape. The venturi effect caused by certain narrow gorges when mist is forced through them by orographic, anabatic and catabatic winds can lead to abnormally high localised rainfall (Hahn 2002; Matthews 1991). The areas just below the escarpment crest, where atmospheric moisture can be trapped most effectively against the south-facing escarpment, generally yield the highest precipitation (Matthews 1991). 
The diversity of rainfall in the study area is reflected in the long-term average rainfall recorded for three farms, namely Ventersdorp, Hanglip and Schyffontein with annual rainfall figures of $585 \mathrm{~mm}, 774 \mathrm{~mm}$ and $835 \mathrm{~mm}$ respectively (South African Weather Bureau 2004). Apart from the spatial variation in rainfall, the area reveals a high temporal variation in recorded rainfall (Geldenhuys \& Murray 1993). Mean annual rainfall for Makhado fluctuated between $571 \mathrm{~mm}$ for the period 1965-1971 and $1027 \mathrm{~mm}$ for the period 1979-1988 (South African Weather Bureau 2004). In addition to these rainfall figures, the amount of precipitation as a result of mist can be substantial (Schutte 1971). In the higher lying areas of the KwaZulu-Natal Drakensberg, for example, the orographic fog contribution at $1800 \mathrm{~m}$ altitude is an additional $403 \mathrm{~mm}$ per annum, which amounts to one third of the mean annual precipitation (Matthews 1991). As with many mountainous areas, the daily weather of the higher altitude crests and summits of the Soutpansberg is very unpredictable; it fluctuates between extremes within a matter of hours.

The higher lying crests and ridges within the mistbelt are exposed to strong winds. During the summer months these winds carry moisture in from the Indian Ocean, creating a seasonal mistbelt, which gives rise to an abundance of rock and bark lichens and bryophytes. The combination of frequent orographic rain and mist during the summer months leaves the available soil drenched, and sometimes flooded for extended periods. During the extended dry season the prevailing winds are dry, causing dehydration and desiccation of the soils and vegetation (Hahn 2002). These extreme and fluctuating environmental conditions have led to specialisation among the plants and may explain the relatively high level of endemisity within this vegetation type (Hahn 2002).

\section{Soutpansberg Centre of Biological Diversity}

The Soutpansberg-Blouberg region has been recognised as a Centre of Endemism by Van Wyk and Smith (2001). However, little ecological knowledge of the area exists (Anderson 2001, Berger et al. 2003). Some floristic surveys conducted by Hahn (1994, 1996 1997, 1999, 2002), Stirton (1982), Obermeyer et al. (1937) and Van Wyk $(1984,1996)$ indicated that the Soutpansberg Centre of Endemism is exceptionally diverse and species-rich for its size (Van Wyk \& Smith 2001). The conservation value of this centre lies in its unique ability to house a wide variety of floristic elements from the surrounding floristic regions (Hahn 2002). The region is an outstanding centre of plant diversity, with approximately 2 500-3 000 recorded vascular plant taxa (Hahn 1997). According to Van Wyk and Smith (2001) $41 \%$ of genera and $68 \%$ of families occurring in southern Africa are represented within the Soutpansberg Centre of Endemism. Altogether 595 specific and infra-specific trees and shrubs are known from the Soutpansberg, amounting to one third of all the known tree species in the entire southern Africa region (Hahn 1994, 1997, 2003). This constitutes one of the highest tree counts in southern Africa (Hahn 1997). The Kruger National Park, which covers an area of two million hectares, contains approximately 380 tree species (Van Wyk 1994), whereas 321 tree species have been recoded by Hahn (2002) in an area of only 2000 hectares within the SC. Trees and shrubs accounts for approximately $24 \%$ of the vascular plants of the Soutpansberg and play an important role in the species composition, vegetation structure and relative dominance within the different plant communities.

More than 500 bird species have been recorded throughout the Soutpansberg Mountain Range, amounting to approximately $56 \%$ of the recorded species for the entire southern Africa (Harrison et al. 1997; Hockey et al. 2005). The Soutpansberg and its surroundings have some unique reptile habitats, and seven endemic species are found there (Branch 1988). A total of 46 spider families, 110 genera and 130 species have been recorded in the SC on the farm Lejuma $\left(<50 \mathrm{~km}^{2}\right)$, which represent $70 \%$ of the families, $26 \%$ of the genera and $5 \%$ of the species recorded for South Africa (Foord et al. 2002, 2003). The high biological diversity of the Soutpansberg and Blouberg can possibly be attributed to the fact that the mountain range acts as a refuge in times of environmental flux (Hahn 2003).

In the light of the high diversity recorded for the BloubergSoutpansberg expanse, it is proposed that the region be given the status of the Soutpansberg Centre of Biological Diversity (SCBD) in addition to its recognised status as the Soutpansberg Centre of Plant Endemism. There are currently efforts underway to create a biosphere reserve in this area, which will include the SC and BNR (Hahn in prep.).

\section{Aims of the study}

This study is a first attempt at understanding the complex ecological patterns and processes observed within the SCBD. It is concerned with the phytosociology and synecology of the SC and BNR. The aim is to identify the different plant communities and to investigate the interrelationships between plant communities and their physical and biological environments. In an attempt to create a holistic image and to explain the macro-ecology of the region, disciplines such as climatology, geology, pedology, physical geography, history and anthropology are drawn upon and integrated. This study provides a first approximation of the vegetation and proposes nine MVT's for the study area. It aims to define and describe the characteristics of these MVT's within the context of the SC and BNR. This will assist scientists, conservationists and land-use planners when future projects are conducted within the surrounding areas. These plant communities from the SC and BNR will serve as reference sites with which to compare proposed development sites from the surrounding unprotected areas. Environmentally sound development is the ethical responsibility to base decisions regarding resourse utilisation on all available information in order to make holistical and long term sense (Siebert 2001). This can be achieved if basic information, such as this account, is actively drawn upon during the planning phases of development and the management of natural resources.

\section{METHODS}

Aerial photographs (scale 1:50 000) were used to stratify the study area into physiographic-physiognomic units. A total of 466 sample plots were randomly placed within each of these stratified units. The sample plot size was set at $400 \mathrm{~m}^{2}$ in accordance with vegetation studies elsewhere in the semiarid environments of southern Africa (Siebert 2001). The cover-abundance value for every species recorded within each sample plot was assessed according to the Braun-Blanquet cover-abundance scale (Mueller-Dombois \& Ellenberg 1974). The taxon names conform to those of Germishuizen and Meyer (2003). Environmental data include soil type, aspect, slope, surface rock cover and disturbance to the soil and vegetation.

The data set, containing 846 specific and infra- specific taxa, was entered into a vegetation database created in TURBOVEG (Hennekens \& Schamineé 2001). A first approximation of the main communities was arrived at by applying the TWINSPAN classification program (Hill 1979a) to the floristic data, and subsequent refinement of the classification was achieved by applying Braun-Blanquet procedures (Bredenkamp et al. 1989; Fuls et al. 1993; Kent \& Coker 1996; Siebert et al. 2003; Van Staden \& Bredenkamp 2006).

A synoptic table was constructed to represent the major groups defined by the TWINSPAN classification (Table 1). Refinement of the synoptic table was done with Braun-Blanquet procedures (Bredenkamp et al. 1989; Fuls et al. 1993; Van Staden 


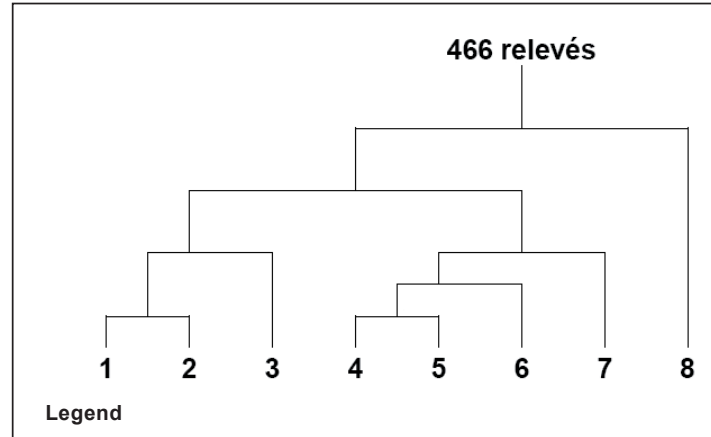

\begin{tabular}{|c|l|}
\hline 1 & $\begin{array}{l}\text { Eragrostis lehmanniana-Sclerocarya birrea Blouberg Northern } \\
\text { Plains Bushveld }\end{array}$ \\
\hline 2 & $\begin{array}{l}\text { Euclea divinorum-Acacia tortilis Blouberg Southern Plains } \\
\text { Bushveld }\end{array}$ \\
\hline 3 & $\begin{array}{l}\text { Englerophytum magalismontanum-Combretum molle Blouberg } \\
\text { Mountain Bushveld }\end{array}$ \\
\hline 4 & $\begin{array}{l}\text { Adansonia digitata-Acacia nigrescens Soutpansberg Arid } \\
\text { Northern Bushveld }\end{array}$ \\
\hline 5 & $\begin{array}{l}\text { Catha edulis-Flueggia virosa Soutpansberg Moist Mountain } \\
\text { Thickets }\end{array}$ \\
\hline 6 & $\begin{array}{l}\text { Diplorhynchus condylocarpon-Burkea africana Soutpansberg } \\
\text { Leached Sandveld }\end{array}$ \\
\hline 7 & $\begin{array}{l}\text { Rhus rigida-Rhus magalismontanum Soutpansberg Mistbelt } \\
\text { Vegetation }\end{array}$ \\
\hline 8 & $\begin{array}{l}\text { Xymalos monospora-Rhus chirendensis Soutpansberg Forest } \\
\text { Vegetation }\end{array}$ \\
\hline
\end{tabular}

\section{FIGURE 3}

A dendrogram showing the hierarchical divisions created by the TWINSPAN (Two Way INdicator SPecies ANalysis) computer software package (Hill 1979a)

\& Bredenkamp 2006). The synoptic table comprises species in each of the identified MVT's on constancy values of $20 \%$ ordinal scale (I-V). Only species with a minimum constancy value of $20 \%$ (II), in any of the given MVT's were included in the table. All the excluded taxa will be included into detailed phytosociological tables of subsequent papers that will focus on the individual major vegetation types of the SC and BNR.

The ordination algorithm DEtrended CORrespondence ANAlysis (DECORANA) (Hill 1979b) was applied, using the computer software package PC-ORD (McCune \& Mefford 1999), to determine gradients in vegetation and the relationship between these plant communities and the physical environment. Results are depicted on scatter diagrams.

\section{RESULTS}

Eight MVT's were identified for the SC and the BNR. These represent the Forest Biome, Grassland Biome, Savanna Biome and some azonal plant communities.

A dendrogram (Fig. 3) of the hierarchical classification was produced with the software package TWINSPAN (Hill 1979a). The first division separated the Xymalos monospora-Rhus chirendensis Soutpansberg Forest Vegetation from the savanna and grassland vegetation types. The second division separated the vegetation of the SC from that of the BNR. A third division split off the Rhus rigida var. rigida-Rhus magalismontanum subsp. coddii Soutpansberg Mistbelt Vegetation from the SC-cluster, and split off the Blouberg Plains Bushveld from the Blouberg Mountain Bushveld. Further divisions separated the Blouberg Northern Plains Bushveld from the Blouberg Southern Plains Bushveld, while the vegetation of the SC was divided into Adansonia digitata-Acacia nigrescens Soutpansberg Arid Northern Bushveld, Catha edulis-Flueggia virosa Soutpansberg Moist Mountain Thickets and Diplorhynchus condylocarponBurkea africana Soutpansberg Leached Sandveld.
The dataset was classified into different plant communities based on diagnostic species. Strong emphasis was placed on long-lived perennial species for the purpose of community description and syntaxonomy. These species were specifically chosen to ensure relatively long-term predictability regarding effective plant community identification by future fieldworkers and managers. Due to the fleeting existence and unpredictable appearance of annual and weak perennial species within communities of arid and semi-arid ecosystems it was decided to treat such species as the more temporary and fluctuating component within the vegetation of these event-driven systems (Westoby et al. 1989).

The floristic composition of the eight major vegetation types is given in a synoptic table (Table 1). These MVT's are discussed below.

\section{Description of the Major Vegetation Types}

1. Eragrostis lehmanniana var. lehmanniana-Sclerocarya birrea subsp. caffra Blouberg Northern Plains Bushveld Major Vegetation Type

The Eragrostis lehmanniana var. lehmanniana-Sclerocarya birrea subsp. caffra Blouberg Northern Plains Bushveld MVT occurs on the northern foot slopes and plains of the Blouberg Nature Reserve. The terrain is generally flat, with a maximum incline of three degrees. This MVT is associated with the Hutton Soil Form (MacVicar et al. 1991) of the Ae Land Type derived from alluvium on sandstone of the Wyllies Poort Geological Formation (Botha 2004a; Patterson \& Ross 2004a).

The diagnostic species for this group are presented in species group A (Table 1). This group includes the woody species Spirostachys africana. Diagnostic grass species include Eragrostis lehmanniana var. lehmanniana, Tragus berteronianus, Dactyloctenium aegyptium and Pogonarthria squarrosa. This diagnostic group contains numerous herbaceous species such as Phyllanthus burchellii, Indigofera species, Limeum fenestratum, Erythrophleum africanum, Bulbostylis hispidula subsp. pyriformis, Ruellia species, Indigofera rhytidocarpa, Chamaecrista absus, Hermannia grisea, Tragia minor, Corchorus species, Talinum crispatulum, Monechma divaricatum, Zornia species, Blepharis subvolubilis, Limeum viscosum, Leucas sexdentata, Secamone parvifolia, Chamaecrista biensis and Asparagus exuvialis forma exuvialis.

Dominant woody species of this MVT include Spirostachys africana (Species Group A), Commiphora species, Grewia flavescens var. flavescens (Species Group F), Boscia albitrunca var. albitrunca, Cissus cornifolia, Combretum apiculatum subsp. apiculatum, Combretum mossambicense, Commiphora mollis, Grewia bicolor (Species Group H), Acacia nigrescens, Dichrostachys cinerea subsp. africana, Ehretia rigida, Markhamia zanzibarica and Sclerocarya birrea subsp. caffra (Species Group L). Dominant grass species include Eragrostis lehmanniana var. lehmanniana (Species Group A), Eragrostis rigidior, Urochloa mosambicensis (Species Group L), Digitaria eriantha, Panicum maximum and Aristida stipitata subsp. graciliflora (Species Group P). Prominent herbaceous species include Phyllanthus burchellii (Species Group A), Acanthospermum species, Arctotis species, Aristida congesta subsp. congesta, Evolvulus alsinoides, Hibiscus calyphyllus, Hibiscus praeteritus, Sida ovata (Species Group C), Cyperus angolensis (Species Group F), Solanum panduriforme (Species Group L) and Waltheria indica (Species Group P).

The vegetation of the Eragrostis lehmanniana var. lehmannianaSclerocaryabirrea subsp.caffra Blouberg Northern Plains Bushveld MVT shows some floristic affinities with the Acacia melliferaEragrostis lehmanniana vegetation class, informally described by Smit (2000) as part of the Eastern Kalahari Thornveld. Shared prominent species within the Blouberg Northern Plains Bushveld and the Acacia mellifera-Eragrostis lehmanniana 
TABLE 1

Synoptic table of the Major Vegetation Types of the Soutpansberg Conservancy and the Blouberg Nature Reserve

\begin{tabular}{|c|c|c|c|c|c|c|c|c|}
\hline VEGETATION TYPE & 1 & 2 & 3 & 4 & 5 & 6 & 7 & 8 \\
\hline NUMBER OF RELEVÉS & 98 & 84 & 16 & 65 & 20 & 50 & 33 & 70 \\
\hline \multicolumn{9}{|l|}{ SPECIES GROUP A } \\
\hline \multicolumn{9}{|c|}{$\begin{array}{l}\text { Diagnostic spp for the Eragrostis lehmanniana var. Iehmanniana- } \\
\text { Sclerocarya birrea subsp. caffra Blouberg Northern Plains Bushveld }\end{array}$} \\
\hline Eragrostis lehmanniana var. lehmanniana & v & I & I & I & & & & \\
\hline Phyllanthus burchellii & IV & & & & & & & \\
\hline Indigofera sp. & III & I & I & & & & & \\
\hline Leucas sexdentataw & III & & & & & & & \\
\hline Limeum fenestratum & III & & & & & & & \\
\hline Erythrophleum africanum & III & & & & & & & \\
\hline Bulbostylis hispidula subsp. pyriformis & III & & I & I & & & I & \\
\hline Ruellia sp. & II & & & & & & & \\
\hline Indigofera rhytidocarpa & II & & & & & & & \\
\hline Chamaecrista absus & II & & & & & & & \\
\hline Hermannia grisea & II & & & & & & & \\
\hline Tragus berteronianus & II & & & & & & & \\
\hline Tragia minor & II & & & & & & & \\
\hline Corchorus sp. & II & I & & & & & & \\
\hline Talinum crispatulum & II & & & & & & & \\
\hline Monechma divaricatum & II & & & & & & & \\
\hline Dactyloctenium aegyptium & II & & & & & & & \\
\hline Zornia sp. & II & & & & & & & \\
\hline Pogonarthria squarrosa & II & & & & & & & \\
\hline Blepharis subvolubilis & II & & & I & & & & \\
\hline Limeum viscosum & II & & & & & & & \\
\hline Secamone parvifolia & II & I & & & & & & \\
\hline Spirostachys africana & II & & & & & & & \\
\hline Chamaecrista biensis & II & & & & & & & \\
\hline Asparagus exuvialis fo. exuvialis & II & & & & & & & \\
\hline
\end{tabular}

SPECIES GROUP B

Diagnostic spp for the Euclea divinorum-Acacia tortilis Blouberg Southern Plains Bushveld

\begin{tabular}{|c|c|c|c|c|}
\hline Lepidagathis scabra & I & v & & \\
\hline Rhinacanthus xerophilus & & III & & \\
\hline Melhania prostrata & & III & I & $\mathbf{I}$ \\
\hline Rhus leptodictya & 1 & II & I & I \\
\hline Enteropogon macrostachyus & & II & & \\
\hline Combretum imberbe & & II & & $\mathbf{I}$ \\
\hline Schotia brachypetala & & II & I & I \\
\hline Aristida congesta subsp. barbicollis & & II & I & I \\
\hline Corbichonia decumbens & & II & & \\
\hline \multicolumn{5}{|l|}{ SPECIES GROUP C } \\
\hline Hibiscus praeteritus & $\mathbf{v}$ & IV & & \\
\hline Acanthospermum sp. & $\mathbf{v}$ & II & & \\
\hline Evolvulus alsinoides & IV & II & & \\
\hline Melhania forbesii & IV & II & & \\
\hline Aristida congesta subsp. congesta & III & II & I & \\
\hline Hibiscus calyphyllus & III & $\mathbf{v}$ & & \\
\hline Sida ovata & III & II & I & \\
\hline Blepharis integrifolia & III & II & I & \\
\hline
\end{tabular}

TABLE 1 (CONT...)

\begin{tabular}{l|llllllll}
\hline VEGETATION TYPE & 1 & 2 & 3 & 4 & 5 & 6 & 7 & 8 \\
\hline NUMBER OF RELEVÉS & 98 & 84 & 16 & 65 & 20 & 50 & 33 & 70 \\
\hline Lantana rugosa & II & IV & I & & & & & \\
Acacia tortilis W. heteracantha & II & III & & I & I & & & \\
Aristida adscensionis & II & III & & & & & \\
Arctotis sp. & II & II & & & & & & \\
Acalypha indica & II & II & & & & & & \\
Philenoptera violacea & II & II & & I & & & & \\
Ocimum gratissimum subsp. gratissimum & II & II & & & & & &
\end{tabular}

SPECIES GROUP D

Diagnostic spp for the Englerophytum magalismontanum-Combretum molle Blouberg Mountain Bushveld

\begin{tabular}{|c|c|c|c|}
\hline \multicolumn{3}{|l|}{ Loudetia filifolia } & IV \\
\hline Rhynchosia vendae & & $\mathbf{I}$ & III \\
\hline \multicolumn{3}{|l|}{ Trichoneura grandiglumis } & III \\
\hline Aristida sp. & & I & III \\
\hline Combretum zeyheri & & $\mathbf{I}$ & II \\
\hline Elephantorrhiza sp. & & & II \\
\hline Dalechampia sp. & & & II \\
\hline Tricliceras schinzii & & & II \\
\hline \multicolumn{4}{|l|}{ SPECIES GROUP E } \\
\hline \multirow{2}{*}{\multicolumn{2}{|c|}{$\begin{array}{l}\text { Cheilanthes involuta } \\
\text { Tephrosia purpurea }\end{array}$}} & & III \\
\hline & & III & IV \\
\hline \multicolumn{4}{|l|}{ SPECIES GROUP F } \\
\hline Cyperus angolensis & III & III & III \\
\hline Commiphora sp. & IV & II & III \\
\hline Grewia flavescens var. flavescens & IV & IV & II \\
\hline Eragrostis biflora & II & II & II \\
\hline
\end{tabular}

SPECIES GROUP G

Diagnostic spp for the Adansonia digitata-Acacia nigrescens Soutpansberg Arid Northern

\begin{tabular}{|c|c|c|c|c|}
\hline Tribulus terrestris & & & III & \\
\hline Grewia hexamita & & & III & \\
\hline Commiphora glandulosa & & & III & \\
\hline Blepharis diversispina & & & III & \\
\hline Adansonia digitata & & & II & \\
\hline Grewia flava & & I & II & \\
\hline Grewia subspathulata & & & II & \\
\hline Cordia monoica & & & II & 1 \\
\hline Kirkia acuminata & & I & II & \\
\hline Maerua parvifolia & & & II & \\
\hline Terminalia prunioides & I & & II & \\
\hline Maerua edulis & & & II & I \\
\hline Commiphora tenuipetiolata & & & II & \\
\hline Tephrosia macropoda & & & II & \\
\hline Solanum lichtensteinii & & & II & I \\
\hline Cassia abbreviata & I & & II & \\
\hline Cleome angustifolia subsp. petersiana & & & II & \\
\hline Ochna inermis & & & II & I \\
\hline Lannea schweinfurthii & I & & II & \\
\hline Ledebouria apertiflora & & & II & \\
\hline
\end{tabular}


TABLE 1 (CONT...)

\begin{tabular}{|c|c|c|c|c|c|c|c|c|}
\hline VEGETATION TYPE & 1 & 2 & 3 & 4 & 5 & 6 & 7 & 8 \\
\hline NUMBER OF RELEVÉS & 98 & 84 & 16 & 65 & 20 & 50 & 33 & 70 \\
\hline Commiphora viminea & & & & II & & & & \\
\hline Sterculia rogersii & & I & & II & & & & \\
\hline Grewia villosa & & & & II & & & & \\
\hline Chamaecrista mimosoides & & & & II & & I & I & \\
\hline Boscia foetida subsp. rehmanniana & & & & II & & & & \\
\hline Sansevieria aethiopica & & & & II & & & & \\
\hline Heliotropium steudneri & I & I & & II & I & & & \\
\hline Commiphora africana var. africana & & & & II & & & & \\
\hline \multicolumn{9}{|l|}{ SPECIES GROUP H } \\
\hline Commiphora mollis & IV & II & & IV & & & & \\
\hline Combretum apiculatum subsp. apiculatum & III & III & I & III & & I & & \\
\hline Boscia albitrunca var. albitrunca & III & II & & II & & & & \\
\hline Grewia bicolor var. bicolor & III & III & & II & I & I & & \\
\hline Cissus cornifolia & II & II & II & II & & & & \\
\hline Combretum mossambicense & III & & & II & & & & \\
\hline Pristimera longipitiolata & II & I & & II & I & & & \\
\hline
\end{tabular}

\section{SPECIES GROUP I}

Diagnostic spp for the Catha edulis-Flueggia virosa Soutpansberg Moist Mountain Thickets

\begin{tabular}{|c|c|c|c|c|c|}
\hline \multicolumn{4}{|l|}{ Grewia occidentalis var. occidentalis } & IV & \\
\hline Dovyalis zeyheri & & & & IV & \\
\hline Acalypha glabrata & & & I & IV & \\
\hline Dombeya rotundifolia var. rotundifolia & I & & & IV & 1 \\
\hline Catha edulis & & & & IV & \\
\hline Rhus pentheri & & & & III & 1 \\
\hline Carissa edulis & & & & III & \\
\hline Rhoicissus tridentata subsp. tridentata & & & & III & I \\
\hline Senna petersiana & $\mathbf{I}$ & & I & III & \\
\hline Diospyros lycioides & & 1 & & III & 1 \\
\hline Berchemia zeyheri & & & & III & \\
\hline Dovyalis caffra & & & & II & \\
\hline Brachiaria deflexa & & & & II & \\
\hline Capparis tomentosa & & & & II & \\
\hline Euphorbia ingens & 1 & & & II & \\
\hline Acacia ataxacantha & I & & & II & I \\
\hline Euclea undulata & & & 1 & II & \\
\hline Pavetta schumanniana & & & & II & 1 \\
\hline Acacia rehmanniana & & & & II & \\
\hline Commelina benghalensis & & & & II & 1 \\
\hline Gymnosporia senegalensis & & & & II & \\
\hline Acokanthera oppositifolia & & & & II & \\
\hline Mystroxylon aethiopicum subsp. schlechteri & & & I & II & \\
\hline Rhus pyroides & & & & II & \\
\hline Bridelia mollis & I & I & & II & \\
\hline Clerodendrum glabrum var. glabrum & & & & II & \\
\hline Ekebergia capensis & & & & II & \\
\hline Allophylus africanus var. africanus & & & & II & \\
\hline Tarchonanthus camphoratus & & & & II & \\
\hline Maerua caffra & & & & II & \\
\hline Coddia rudis & & & & II & 1 \\
\hline
\end{tabular}

TABLE 1 (CONT...)

\begin{tabular}{|c|c|c|c|c|c|c|c|c|}
\hline VEGETATION TYPE & 1 & 2 & 3 & 4 & 5 & 6 & 7 & 8 \\
\hline NUMBER OF RELEVÉS & 98 & 84 & 16 & 65 & 20 & 50 & 33 & 70 \\
\hline Lippia javanica & & & & & II & & I & \\
\hline Jasminum multipartitum & & & & & II & 1 & I & \\
\hline Combretum hereroense & I & I & & I & II & & & \\
\hline Eragrostis superba & & & & & II & & I & \\
\hline Panicum deustum & & I & & 1 & II & & & \\
\hline Acacia caffra & & & & & II & & & \\
\hline Setaria megaphylla & & & & & II & & & \\
\hline Christella guenziana & & & & & II & & & \\
\hline Euclea crispa subsp. crispa & & & & & II & & & \\
\hline Cyperus albostriatus & & & & & II & & I & \\
\hline Olea europaea subsp. africana & & & & & II & & & \\
\hline Bridelia micrantha & & & & & II & & & \\
\hline Buddleja saligna & & & & & II & & & \\
\hline Solanum tettense var. renschii & & & & I & II & & & \\
\hline Cussonia natalensis & & & & & II & & & \\
\hline Ficus sycomorus subsp. sycomorus & & & & & II & & & \\
\hline Dicliptera heterostegia & & & & I & II & & & \\
\hline Scolopia zeyheri & & & & & II & & & \\
\hline Canthium inerme & & & & & II & & & \\
\hline Buddleja salviifolia & & & & & II & & & \\
\hline Podocarpus falcatus & & & & & II & & & \\
\hline Pyrenacantha grandiflorus & & & & & II & & & \\
\hline Aloe greatheadii var. greatheadii & & & & & II & & I & \\
\hline Canthium mundianum & & & & & II & I & & \\
\hline Bothriochloa insculpta & & & & & II & & & \\
\hline Ficus sur & & & & & II & & I & \\
\hline Sansevieria hyacinthoides & & & & I & II & & & \\
\hline Cyperus sphaerospermus & & & & & II & & I & \\
\hline Bulbostylis burchellii & & & & & II & & I & \\
\hline Syzygium cordatum & & & & & II & & & \\
\hline Aloe marlothii subsp. marlothii & & & & & II & & & \\
\hline Barleria gueinzii & & & & & II & & & \\
\hline Pavetta eylesii & & & & & II & & & \\
\hline Capparis fascicularis var. fascicularis & & & & & II & & & \\
\hline Acacia gerrardii var. gerrardii & & & & & II & & & \\
\hline Justicia flava & 1 & & & 1 & II & & & \\
\hline \multicolumn{9}{|l|}{ SPECIES GROUP J } \\
\hline Plectroniella armata & & & & II & III & I & & \\
\hline Hibiscus meyeri & & & & III & II & & & \\
\hline Peltophorum africanum & & I & & II & III & I & & \\
\hline Ximenia caffra var. caffra & & & & II & II & I & & \\
\hline Gossypium herbaceum subsp. africanum & & & & II & II & & & \\
\hline \multicolumn{9}{|l|}{ SPECIES GROUP $K$} \\
\hline Euclea divinorum & & $\mathbf{v}$ & & II & II & & & \\
\hline Flueggia virosa & I & III & & II & IV & & & \\
\hline Ximenia americana var. microphylla & & II & & II & II & & & \\
\hline Heteropogon contortus & 1 & II & & II & II & & I & \\
\hline Themeda triandra & & II & II & I & III & & I & \\
\hline Acacia karroo & & II & & & $\mathbf{v}$ & & & \\
\hline
\end{tabular}


TABLE 1 (CONT...)

\begin{tabular}{|c|c|c|c|c|c|c|c|c|}
\hline VEGETATION TYPE & 1 & 2 & 3 & 4 & 5 & 6 & 7 & 8 \\
\hline NUMBER OF RELEVÉS & 98 & 84 & 16 & 65 & 20 & 50 & 33 & 70 \\
\hline Gymnosporia buxifolia & I & II & & I & III & & & \\
\hline Pappea capensis & & II & I & I & II & & & \\
\hline Acacia nilotica & & II & & 1 & II & & & \\
\hline \multicolumn{9}{|l|}{ SPECIES GROUP L } \\
\hline Dichrostachys cinerea subsp. africana & IV & IV & & IV & III & & & \\
\hline Solanum panduriforme & III & IV & II & I & II & & & \\
\hline Acacia nigrescens & II & IV & & IV & II & I & & \\
\hline Urochloa mosambicensis & III & II & & & II & & & \\
\hline Sclerocarya birrea subsp. caffra & IV & II & & II & II & & & \\
\hline Eragrostis rigidior & III & III & & I & II & & & \\
\hline Grewia monticola & III & IV & & III & II & 1 & & \\
\hline Ehretia rigida & II & IV & & II & III & & & \\
\hline Markhamia zanzibarica & IV & $\mathbf{I}$ & & 1 & II & & & \\
\hline
\end{tabular}

\section{SPECIES GROUP M}

Diagnostic spp for the Diplorhynchus condylocarpon-Burkea africana Soutpansberg Leached Sandveld

Centropodia glauca

Elephantorrhiza burkei

Diplorhynchus condylocarpon

Ochna pulchra

Hexalobus monopetalus var. monopetalus

Grewia retinervis

Ipomoea albivenia

Strychnos pungens

Eragrostis pallens

Ozoroa paniculosa var. salicina

Schizachyrium jeffreysii

Selaginella dregei

Euphorbia aeruginosa

Aloe angelica

Pterocarpus angolensis

Garcinia livingstonei

Eragrostis gummiflua

Euphorbia zoutpansbergensis

Commiphora marlothii

Ficus abutilifolia

Euphorbia cooperi

Cineraria parvifolia

Artabotrys brachypetalus

Portulaca kermesina

Aristida canescens subsp. ramosa

Tephrosia longipes

Aristida diffusa subsp. burkei

Indigofera cryptantha var. cryptantha

Ficus tettensis

Adenia spinosa

Orthosiphon labiatus

Isoglossa hypoestiflora

Anacampseros subnuda

|III

III

III

III
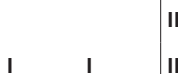

(I)

\begin{tabular}{l|l} 
I II \\
II
\end{tabular}

II

(II

II

TABLE 1 (CONT...)

\begin{tabular}{|c|c|c|c|c|c|c|c|c|}
\hline VEGETATION TYPE & 1 & 2 & 3 & 4 & 5 & 6 & 7 & 8 \\
\hline NUMBER OF RELEVÉS & 98 & 84 & 16 & 65 & 20 & 50 & 33 & 70 \\
\hline Asparagus laricinus & & & & & & II & & \\
\hline Loudetia flavida & & & & & & II & & \\
\hline Androstachys johnsonii & & & & & & II & & \\
\hline Dicoma montana & & & & & & II & & \\
\hline \multicolumn{9}{|l|}{ SPECIES GROUP N } \\
\hline Tricalysia junodii var. kirkii & & & & II & & II & & \\
\hline Euclea natalensis & I & & & II & II & II & & \\
\hline \multicolumn{9}{|l|}{ SPECIES GROUP O } \\
\hline Pseudolachnostylis maprouneifolia & & $\mathrm{I}$ & IV & 1 & & IV & & \\
\hline Burkea africana & & & III & & & IV & & \\
\hline \multicolumn{9}{|l|}{ SPECIES GROUP P } \\
\hline Panicum maximum & $\mathbf{v}$ & $\mathbf{v}$ & IV & III & IV & II & & \\
\hline Digitaria eriantha & IV & I & IV & 1 & I & II & I & \\
\hline Ziziphus mucronata & II & III & I & II & $\mathbf{v}$ & II & & \\
\hline Strychnos madagascariensis & II & I & IV & II & & III & & \\
\hline Enneapogon cenchroides & III & III & II & II & & III & 1 & \\
\hline Waltheria indica & IV & II & II & II & & II & & \\
\hline Schmidtia pappophoroides & III & I & II & III & & II & & \\
\hline Terminalia sericea & II & I & II & ॥ & & III & & \\
\hline Aristida stipitata subsp. graciliflora & III & & & II & & II & & \\
\hline Stipagrostis uniplumis var. uniplumis & II & & I & II & & II & & \\
\hline
\end{tabular}

SPECIES GROUP $Q$

Diagnostic spp for the Rhus rigida var rigida-Rhus magalismontanum subsp. coddii Soutpansberg Mistbelt Vegetation

Rhus rigida var rigida

Melinis nerviglumis

Helichrysum kraussii

Brachiaria serrata

Cryptolepis cryptolepioides

Parinari capensis subsp. capensis

Fadogia homblei

Coleochloa setifera

Setaria sphacelata var. torta

Rhynchosia monophylla

Olea capensis subsp. enervis

Senecio barbertonicus

Syzygium legatii

Aloe arborescens

Rotheca myricoides

Euclea linearis

Crassula swaziensis

Khadia borealis

Trachypogon spicatus

Rhus tumulicola var. meeuseana

Commelina erecta

Olinia rochetiana

Cyperus obtusiflorus var. obtusiflorus

Combretum moggii

Vernonia natalensis

I $\quad$\begin{tabular}{l|l} 
I IV \\
III \\
III \\
III \\
III \\
III \\
III \\
III \\
II \\
II \\
II \\
II \\
II \\
II \\
II \\
II \\
II \\
II \\
II \\
II \\
II \\
II \\
\hline II \\
\hline II \\
\hline
\end{tabular}


TABLE 1 (CONT...)

\begin{tabular}{|c|c|c|c|c|c|c|c|c|}
\hline VEGETATION TYPE & 1 & 2 & 3 & 4 & 5 & 6 & 7 & 8 \\
\hline NUMBER OF RELEVÉS & 98 & 84 & 16 & 65 & 20 & 50 & 33 & 70 \\
\hline Dicoma anomala & & & I & & & & II & \\
\hline Vangueria soutpansbergensis & & & & & & I & II & \\
\hline Schistostephium crataegifolium & & & & & & & II & \\
\hline Coptosperma supra-axillare & & & & & & & II & \\
\hline Plectranthus neochilus & & & & & & & II & \\
\hline Gnidia cuneata & & & & & & & II & \\
\hline Elionurus muticus & & & & & & & II & \\
\hline Protea caffra subsp. caffra & & & & & & & II & \\
\hline Elephantorrhiza elephantina & & & & & & & II & \\
\hline Tetradenia riparia & & & & & & & II & \\
\hline Wahlenbergia undulata & & & & & & & II & \\
\hline Hypoxis argentea var. argentea & & & & & & & II & \\
\hline Eulophia ensata & & & & & & & II & \\
\hline Pteridium aquilinum & & & & & & & II & \\
\hline Kalanchoe sexangularis var. sexangularis & & & & & & & II & \\
\hline Sarcostemma viminale & & & & I & I & & II & \\
\hline Aristea woodii & & & & & & & II & \\
\hline Apodytes dimidiata subsp. dimidiata & & & & & & & II & \\
\hline Anthospermum we/witschii & & & & & & & II & \\
\hline Vernonia oligocephala & & & & & & & II & \\
\hline Protea roupelliae subsp. roupelliae & & & & & & & II & \\
\hline Pentanisia prunelloides subsp. prunelloides & & & & & & & II & \\
\hline Lopholaena coriifolia & & & & & & & II & \\
\hline Senecio oxyriifolius & & & & & & & II & \\
\hline Coptosperma rhodesiacum & & & & & & & II & \\
\hline Viscum rotundifolium & & & & & & & II & \\
\hline Ekebergia pterophylla & & & & & & & II & \\
\hline Myrsine africana & & & & & & & II & \\
\hline Ipomoea oblongata & & & & & & & II & \\
\hline Helichrysum cerastioides & & & & & & & II & \\
\hline Bulbostylis contexta & & & & & & & II & \\
\hline Hypoxis hemerocallidea & & & & & & & II & \\
\hline Asparagus falcatus & & & & & & I & II & \\
\hline
\end{tabular}

\section{SPECIES GROUP R}

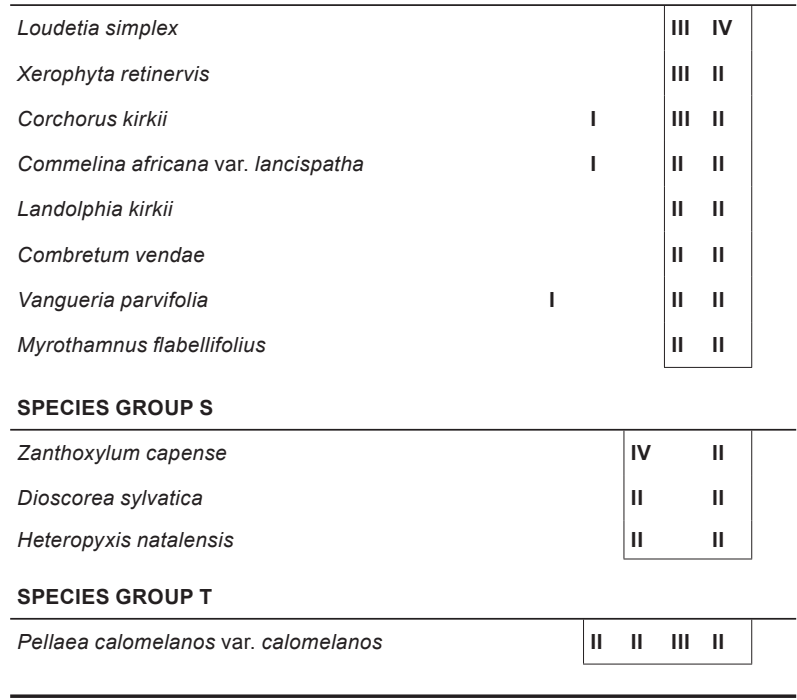

TABLE 1 (CONT...)

\begin{tabular}{|c|c|c|c|c|c|c|c|c|}
\hline VEGETATION TYPE & 1 & 2 & 3 & 4 & 5 & 6 & 7 & 8 \\
\hline NUMBER OF RELEVÉS & 98 & 84 & 16 & 65 & 20 & 50 & 33 & 70 \\
\hline \multicolumn{9}{|l|}{ SPECIES GROUP U } \\
\hline Combretum molle & & & V & & IV & II & III & \\
\hline Vitex rehmannii & & I & IV & & I & III & II & \\
\hline Englerophytum magalismontanum & & & IV & & & I & III & \\
\hline Vangueria infausta subsp. infausta & I & I & III & & II & II & II & \\
\hline Rhus magalismontanum subsp. coddii & & & II & & & & IV & \\
\hline Hyperacanthus amoenus & & & II & & II & II & II & \\
\hline Mimusops zeyheri & & & II & & II & & III & \\
\hline
\end{tabular}

SPECIES GROUP V

Diagnostic spp for the Xymalos monospora-Rhus chirendensis Soutpansberg Forest Vegetation

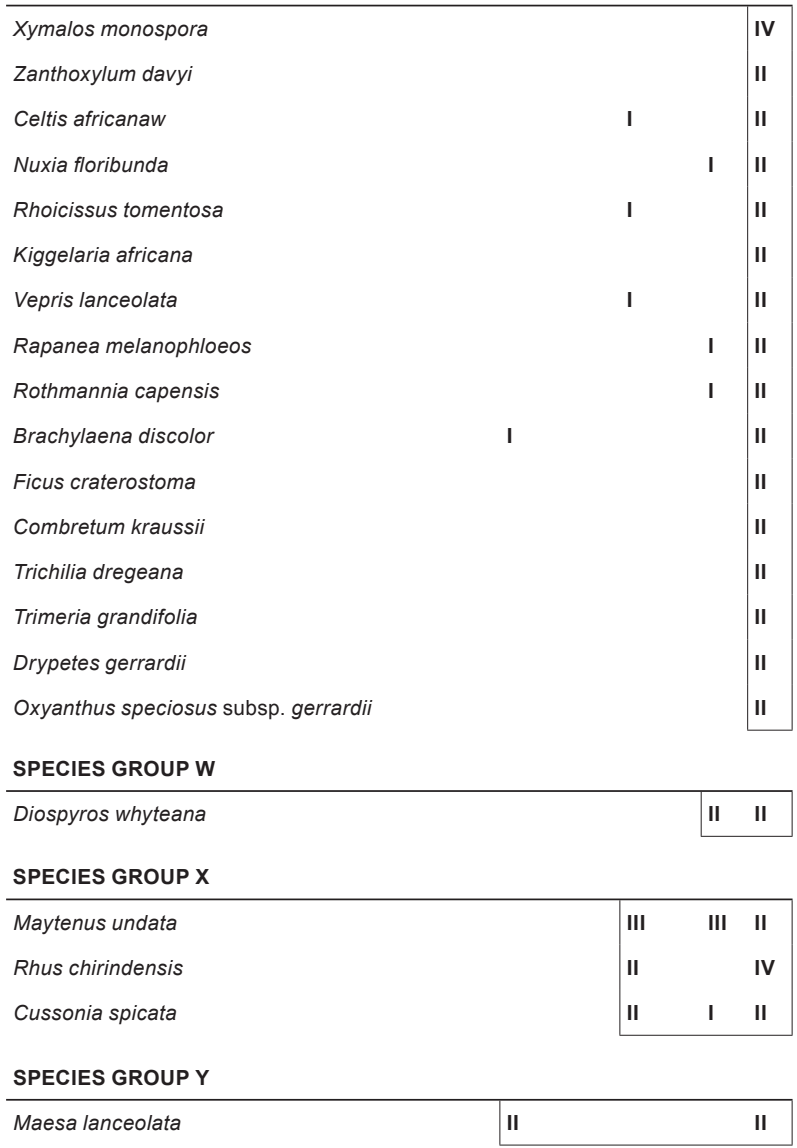

vegetation class include the grasses Eragrostis lehmanniana, Pogonarthria squarrosa and Schmidtia pappophoroides. A shared prominent woody species is Boscia albitrunca. However, these five species all show wide distribution ranges and exhibit wide ecological tolerance and adaptation within the sandveld areas of southern Africa, and are therefore not exclusive to the Blouberg Arid Mountain Bushveld. This major vegetation type shares some of the drought-resistant woody species with the Adansonia digitata-Acacia nigrescens Soutpansberg Arid Northern Bushveld Major Vegetation Type, such as Commiphora mollis, Combretum apiculatum, Boscia albitrunca var. albitrunca, Grewia bicolor, Combretum mossambicense, Commiphora africana, Dichrostachys cinerea subsp. africana and Acacia nigrescens. These species are also commonly found in the Adansonia Mixed Thornveld (14e) (Acocks 1953), the Adansonia digitata-Colophospermum mopane Rugged Veld (Gertenbach 1983) and the Commiphora-Terminalia prunioides community (Louw 1970). 
2. Euclea divinorum-Acacia tortilis Blouberg Southern Plains Bushveld Major Vegetation Type

The Euclea divinorum-Acacia tortilis Blouberg Southern Plains Bushveld MVT is restricted to the plains and foot slopes south of the Blouberg within the BNR. The terrain is generally flat with a slope that varies between one to five degrees. It is predominantly associated with the Hutton Soil Form (MacVicar et al. 1991), derived from alluvium on sandstone of the Ae Land Type from the Wyllies Poort Geological Formation (Botha 2004b; Patterson \& Ross 2004a).

Other Soil Forms associated with this major vegetation type are of less importance. The Oakleaf and Valsrivier Soil Forms (MacVicar et al. 1991) are associated with alluvium, sand and calcrete of the Quaternary Deposits of the Ia Land Type (Botha 2004b). The Glenrosa and Mispah Soil Forms (MacVicar et al. 1991) of the Fc Land Type (Botha 2004b) are associated with basalt of the Letaba Geological Formation in the Lebombo Group-Karoo Sequence, as well as aphibolite and metapelite of the Malala Drift group. The Mispah, Avalon and Hutton Soil Forms (MacVicar et al. 1991) of the Bc Land Type (Botha 2004b) are associated with leucocratic migmatite and gneiss, grey and pink hornblende-biotite gneiss, grey biotite gneiss, minor muscovite-bearing granite, pegmatite and gneiss of the Hout River Gneis Geological Formation, as well as metapelite of the Bandelierkop Complex.

The diagnostic species for this group are presented in species group B (Table 1). The diagnostic woody species characterising the communities of this MVT are Combretum imberbe, Rhus leptodictya and Schotia brachypetala. Diagnostic grass species include Enteropogon macrostachyus and Aristida congesta subsp. barbicollis. Diagnostic herbaceous species within this group are Lepidagathis scabra, Rhinacanthus xerophilus, Melhania prostrata and Corbichonia decumbens.

Dominant woody species of this MVT include Acacia tortilis subsp. heteracantha, Lantana rugosa, Rhus leptodictya (Species Group C), Grewia flavescens var. flavescens (Species Group F), Combretum apiculatum subsp. apiculatum, Grewia bicolor var. bicolor (Species Group H), Acacia nilotica, Euclea divinorum (Species Group K), Acacia nigrescens, Dichrostachys cinerea subsp. africana, Ehretia rigida, Eragrostis rigidior (Species Group L) and Ziziphus mucronata (Species Group P). Dominant grass species include Enteropogon macrostachyus (Species Group B), Aristida congesta subsp. congesta, Aristida adscensionis (Species Group C), Aristida species (Species Group D), Eragrostis rigidior, Urochloa mosambicensis (Species Group L), Enneapogon cenchroides and Panicum maximum (Species Group P). Prominent forbs include Hibiscus praeteritus, Sida ovata, Blepharis integrifolia (Species Group C), Tephrosia purpurea (Species Group E), Cyperus angolensis (Species Group F) and Solanum panduriforme (Species Group L).

The Euclea divinorum-Acacia tortilis Blouberg Southern Plains Bushveld major vegetation type is regarded as a variant of the Rhus leptodictya-Acacia tortilis Bushveld of the Acacietalia rehmannianae-tortilis of the Acacienea nilotico-tortilis of the Panico maximi-Acacietea tortilis, described by Winterbach (1998) and Winterbach et al. (2000) for the north-western savanna of South Africa. Acocks (1953) described similar vegetation as the Knoppiesdoring Veld (13b) of the Other Turf Thornveld (13). It also compares floristically with the Acacia tortilis-Panicum maximum-Ziziphus mucronata major plant community of the Waterberg (Henning 2002). These communities are generally not geographically restricted to a certain part of South Africa, but occur in a patchy distribution where conditions are favourable. Important taxa binding these communities together are Acacia tortilis subsp. heteracantha Rhus leptodictya, Grewia species, Acacia nilotica, Euclea divinorum, Acacia nigrescens,
Dichrostachys cinerea subsp. africana, Ehretia rigida, Eragrostis rigidior, Ziziphus mucronata, Aristida species Eragrostis rigidior, Urochloa mosambicensis and Panicum maximum.

3. Englerophytum magalismontanum-Combretum molle Blouberg Mountain Bushveld Major Vegetation Type

The Englerophytum magalismontanum-Combretum molle Blouberg Mountain Bushveld major vegetation type can be described as the mountain bushveld component of the BNR. This MVT is restricted to the higher lying mountainous terrain of the BNR, ranging from 975-1 $465 \mathrm{~m}$ above sea level. West of the BNR the Blouberg rises to $2051 \mathrm{~m}$ above sea level, where more temperate vegetation types occur (Van Jaarsveld \& Hardy 1991).

Average slopes range from moderate to very steep, with northern, southern and eastern aspects. Soils are generally shallow or skeletal $(<100 \mathrm{~mm})$, and are associated with Mispah and Glenrosa Soil Forms (MacVicar et al. 1991) of the Fa Land Type (Botha 2004a; Patterson \& Ross 2004a). The underlying geology is dominated by pink quartzite and minor conglomerates of the Wyllies Poort Geological Formation of the Soutpansberg Group.

The diagnostic species for this group are presented in species group D (Table 1). Diagnostic woody species include Combretum zeyheri and an Elephantorrhiza species. The diagnostic grass species include Loudetia filifolia, Trichoneura grandiglumis and Aristida species. Diagnostic herbaceous species include Rhynchosia vendae, a Dalechampia species and Tricliceras schinzii.

Dominant woody species of the Englerophytum magalismontanum-Combretum molle Blouberg Mountain Bushveld include Combretum zeyheri, Elephantorrhiza species (Species Group D), Commiphora species (Species Group F), Burkea africana, Pseudolachnostylis maprouneifolia (Species Group O), Strychnos madagascariensis (Species Group P), Combretum molle, Englerophytum magalismontanum, Hyperacanthus amoenus, Mimusops zeyheri, Rhus magalismontanum subsp. coddii, Vangueria infausta subsp. infausta and Vitex rehmannii (Species Group U). Dominant grass species include Aristida species, Loudetia filifolia, Trichoneura grandiglumis (Species Group D), Digitaria eriantha, Enneapogon cenchroides, Panicum maximum and Schmidtia pappophoroides (Species Group P). Prominent herbaceous species include Rhynchosia vendae (Species Group D), Cheilanthes involuta, Tephrosia purpurea (Species Group E), Cyperus angolensis (Species Group F) and Waltheria indica (Species Group P).

The Englerophytum magalismontanum-Combretum molle Blouberg Mountain Bushveld Major Vegetation Type shares floristic elements with the Englerophyto magalismontani-Acacietea caffrae of the Waterberg and Magaliesberg, as described by Winterbach et al. (2000). It shares many of the relatively drought tolerant species associated with the Rhus rigida var. rigida-Rhus magalismontanum subsp. coddii Soutpansberg Cool Mistbelt Major Vegetation Type. It does however lack some of the more mesic species recorded within the mistbelt of the SC. The central variation of the Bankenveld (Acocks 1953) and the Rocky Highveld Grassland of the Grassland Biome (Bredenkamp \& Van Rooyen 1996) share limited floristic and structural elements with the Rhynchosia vendae-Englerophytum magalismontanum Blouberg Moist Mountain Bushveld Major Vegetation Type. Although the vegetation structure of the Diplorhynchus condylocarpon-Englerophytum magalismontanum Rocky Slope community of the Waterberg Biosphere (Henning 2002) is very similar to that of the Blouberg Moist Mountain Bushveld, the floristic composition differs considerably.

\section{Adansonia digitata-Acacia nigrescens Soutpansberg Arid} Northern Bushveld Major Vegetation Type

The Adansonia digitata-Acacia nigrescens Soutpansberg Arid Northern Bushveld major vegetation type is confined to the rain-shadow northern ridges of the SC. Due to the wide range of 
topographic and edaphic conditions found within this MVT, it comprises a complex of very diverse plant communities. Despite the diversity of these plant communities, most are adapted to prolonged water-stress conditions and unpredictable rainfall events. These drought events are likely to be the main driving factors behind the vegetation structure and species composition of the various plant communities within this major vegetation type.

This Soutpansberg Arid Northern Bushveld MVT is associated with the Clovelly Soil Form (MacVicar et al. 1991) of the Ae, Ag, $\mathrm{Ia}, \mathrm{Ib}$, and Fa Land Types derived from sandstone, quartzite and conglomerate of the Wyllies Poort Geological Formation, basalt from the Musekwa Geological Formation, as well as from narrow diabase intrusions or dykes within the Wyllies Poort Geological Formation (Botha 2004a; Patterson \& Ross 2004a). The Ia Land Type in particular is associated with the Aeolian sands (Kalahari sand) covering the Musekwa sediments at the foot of the mountain.

The vegetation structure can generally be described as open woodland (Edwards 1983), with a very sparse field layer. This is especially true during dry cycles, when only the most hardy perennial grass and herbaceous species survive. The woody layer ranges from open along the deeper well-drained sandy soils of the northern plains, to sparse along the shallower clayey foot slopes and around the saltpan.

The diagnostic species for this group are presented in species group G (Table 1). Diagnostic woody species characterising the communities of this MVT along the northern sandy plains include Adansonia digitata, Boscia foetida subsp. rehmanniana, Commiphora slandulosa, Commiphora tenuipetiolata, Cordia monoica, Blepharis diversispina, Grewia flava, Grewia subspathulata, Grewia villosa and Grewia hexamita. Diagnostic woody species along the basaltic-clay foot slopes are Kirkia acuminata, Maerua parvifolia, Maerua edulis, Terminalia prunioides, Cassia abbreviata, Sterculia rogersii and Commiphora viminea. Although Tribulus terrestris was recorded as a relatively strong diagnostic herbaceous species for the group, due to its status as a pioneer annual herbaceous species that dominates disturbed patches, it is not recommended as a reliable indicator species for the Soutpansberg Arid Northern Bushveld. Instead, Sansevieria aethiopica and Ledebouria apertiflora should be seen as more reliable perennial herbaceous indicator species for this broad MVT.

Prominent woody species of this MVT include Grewia hexamita, Commiphora glandulosa, Blepharis diversispina, Adansonia digitata (Species Group G), Commiphora mollis, Combretum apiculatum (Species Group H), Hibiscus meyeri (Species Group J), Acacia nigrescens, Dichrostachys cinerea and Grewia monticola (Species Group L). Although none of the grass species could be labelled as prominent during the time of the surveys, the most dominant grass species included Panicum maximum and Schmidtia pappophoroides (Species Group P). Forbs recorded from the poorly developed field layer are Tephrosia macropoda, Solanum lichtensteinii, Ledebouria apertiflora, Chamaecrista mimosoides, Heliotropium steudneri (Species Group G), Hibiscus meyeri, Gossypium herbaceum subsp. africanum (Species Group J), Waltheria indica (Species Group P) and Pellaea calomelanos var. calomelanos (Species Group T).

The Adansonia digitata-Acacia nigrescens Soutpansberg Arid Northern Bushveld Major Vegetation Type has been described by Acocks (1953) as the Adansonia-Mixed Thornveld variant (14e) of the Arid Sweet Bushveld (14). Due to the scale at which these Veld Types were mapped, Acocks' (1953) description includes more variation along the lower lying microphyllous plains between the Blouberg and Soutpansberg Mountain Ranges and less along the foot slopes of these mountains. The Adansonia-Mixed Thornveld variant (14e) described by Acocks (1953) is therefore very similar to the associations along the northern sandy plains described here under the Soutpansberg Arid Northern Bushveld major vegetation type.

\section{Catha edulis-Flueggia virosa Soutpansberg Moist Mountain} Thickets major vegetation Type

The Catha edulis-Flueggia virosa Moist Mountain Thickets MVT is a mixture of plant communities situated at different altitudes. They are, however, all associated with soils of a high clay content and relatively moist conditions. Even during dry cycles, the moisture-laden air from the south-east ensures at least some orographic rain and moisture during the summer months. These plant communities are primarily confined to the steep southern slopes where igneous rock in the form of basalt and diabase settled after flowing through the cracks and tears in the upper sedimentary rock layers. However, it also contains plant communities associated with the illuvial clays found in some of the valleys cutting through the mountain ridges.

The Moist Mountain Thickets are associated with the Shortlands Soil Sorm (MacVicar et al. 1991) derived from basalt and tuff associated with the Fa Land Type of the Sibasa Geological Formation, as well as from narrow diabase intrusions or dykes associated with the Ib Land Type of the Wyllies Poort Geological Formation (Botha 2004b; Patterson \& Ross 2004b).

The vegetation structure can be described as low, closed thickets (Edwards 1983), with no definite separation between the tree and shrub layers. The woody layer contains a mixture of trees and shrubs, ranging from 1.5-4 m in height. A very high percentage canopy cover $(>80 \%)$ blocks sunlight from reaching the understory and prevents the establishment of a dense field layer.

This major vegetation type is characterised by a high diversity of trees and shrubs. The diagnostic species are presented in species group I (Table 1) and include woody species from relatively moist thickets and wet riverine thickets such as Catha edulis, Grewia occidentalis, Dovyalis zeyheri, Acalypha glabrata, Dombeya rotundifolia, Rhus pentheri, Carissa edulis, Rhoicissus tridentata subsp. tridentata, Senna petersiana, Diospyros lycioides, Berchemia zeyheri, Dovyalis caffra, Capparis tomentosa, Euphorbia ingens, Acacia ataxacantha, Euclea undulata, Pavetta schumanniana, Acacia rehmanniana, Gymnosporia senegalensis, Acokanthera oppositifolia, Mystroxylon aethiopicum subsp. schlechteri, Rhus pyroides, Bridelia mollis, Clerodendrum glabrum var. glabrum, Ekebergia capensis, Allophylus africanus var. africanus, Tarchonanthus camphoratus, Maerua caffra, Coddia rudis, Lippia javanica, Jasminum multipartitum, Combretum hereroense, Acacia caffra, Euclea crispa subsp. crispa, Olea europaea subsp. africana, Bridelia micrantha, Buddleja saligna, Cussonia natalensis, Ficus sycomorus subsp. sycomorus, Scolopia zeyheri, Canthium inerme, Buddleja salviifolia, Podocarpus falcatus, Pyrenacantha grandiflorus, Canthium mundianum, Ficus sur, Syzygium cordatum, Pavetta eylesii, Capparis fascicularis var. fascicularis and Acacia gerrardii var. gerrardii. The recorded diagnostic succulent species listed in species group I are generally widespread throughout South Africa and include Aloe greatheadii var. greatheadii, Sansevieria hyacinthoides and Aloe marlothii subsp. marlothii. Diagnostic grass species recorded from the poorly developed field layer are Brachiaria deflexa, Eragrostis superba, Panicum deustum, Setaria megaphylla and Bothriochloa insculpta. Diagnostic herbaceous species include Commelina benghalensis, Christella guenziana, Cyperus albostriatus, Solanum tettense var. renschii, Dicliptera heterostegia, Cyperus sphaerospermus, Bulbostylis burchellii, Barleria gueinzii and Justicia flava.

Other prominent woody species include Plectroniella armata, Peltophorum africanum (Species Group J), Flueggia virosa subsp. virosa, Acacia karroo, Gymnosporia buxifolia (Species Group K), Ehretia rigida (Species Group L), Ziziphus mucronata (Species Group P), Heteropyxis natalensis, Zanthoxylum capense (Species 
Group S), Combretum molle (Species Group U) and Maytenus undata (Species Group X). Only a few shade-tolerant grass and herbaceous species are prominent within this MVT such as Brachiaria deflexa, Panicum deustum, Setaria megaphylla (Species Group I) and Panicum maximum (Species Group P). The shaded conditions also favour fern species such as Christella gueinziana (Species Group I) and Pellaea calomelanos var. calomelanos (Species Group T).

Acocks (1953) considered this Major Vegetation Type one of the many variants of the "Sourish Mixed Bushveld" and the "North-eastern Mountain Sourveld". Due to its association with the narrow intrusive basalt, tuff and diabase dykes, it is often difficult to map this major vegetation type when dealing with coarse or small-scale vegetation studies. The southernmost ridge of the mountain with its large southern basalt slope is one of the few extensive and mappable areas with this major vegetation type.

The strong mixture of temperate and sub-tropical plant species reflects the gradient of plant communities up the southern slopes of the SC. While those communities along the warm and relatively humid foot slopes are very tropical, the plant communities of clayey depressions along the cooler higher lying areas tend to contain many temperate species. The lower lying sub-tropical plant communities seem to be unique in their species composition and structure. The higher lying temperate communities compare with the Hillside scrub of the Transitional Cymbopogon-Themeda Veld (49) of the Pure Grassveld Types described by Acocks (1953). They share species such as Acacia karroo, Acacia caffra, Grewia occidentalis, Ehretia rigida, Euclea crispa subsp. crispa, Olea europaea subsp. africana, Buddleja saligna, Buddleja salviifolia, Rhus pyroides, Tarchonanthus camphoratus, Diospyros lycioides, Ziziphus mucronata, Dombeya rotundifolia and Cussonia species. These temperate clay communities of the SC also share some floristic links with the riverine woodland Zizipho mucronatae-Acacietum karroo described by Brown (1997). According to Du Preez and Bredenkamp (1991), Bezuidenhout et al. (1994) and Winterbach (1998), the Acacia karroo-dominated vegetation of the southern African grassveld and savanna should be classified as a separate syntaxonomical class. The high-lying communities associated with fine textured clayey soils along the southern slopes of the SC may be classified as part of this proposed class.

6. Diplorhynchus condylocarpon-Burkea africana Soutpansberg Leached Sandveld Major Vegetation Type

The Diplorhynchus condylocarpon-Burkea africana Leached Sandveld MVT is confined to the warmer northern slopes of the mountain, as well as some of the more arid southern slopes along the northernmost ridges of the mountain range, which falls within the rain shadow zone of the mountain.

It is associated with the Mispah and Hutton Soil Forms (MacVicar et al. 1991) derived from sandstone, quartzite and conglomerate associated with the $\mathrm{Ae}, \mathrm{Fa}$, and $\mathrm{Ib}$ Land Types of the Wyllies Poort Geological Formation (Botha 2004a; Patterson \& Ross 2004a). One plant community in particular is associated with deep regic sands of the Namib Soil Form, which is of an Aeolian origin from the Kalahari (Brandl 2002). It also includes the vegetation associated with the almost bare sheets of exposed rock against the warm northern slopes.

The plant communities of this MVT occur on both very shallow and very deep sands of the relatively dry landscapes of the SC. The shallow soils are situated on steep rocky inclines, while the deep sands are associated with relatively high-lying flat plateaus. The combination of the underlying nutrient poor quartzite parent material and the eluviation of silt and clay particles from these well drained sandy soils have left this system extremely nutrient poor.

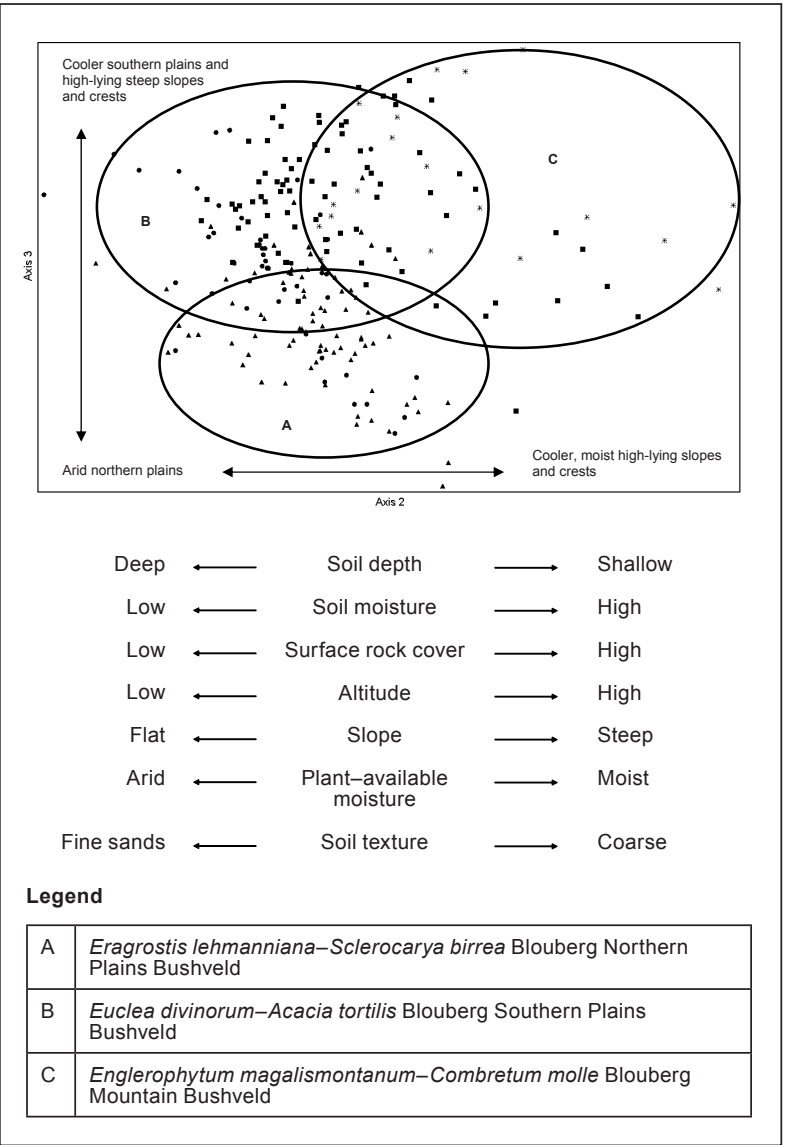

FIGURE 4

Relative positions of relevés along the second and third axes of the DECORANA ordination (Hill 1979b) of the vegetation data from the Blouberg Nature Reserve.

With the exception of the plant communities associated with the rock sheets, the Leached Sandveld communities are relatively poor in plant species diversity. The diagnostic species for this group are presented in Species Group M (Table 1). The Leached Sandveld MVT is characterised by diagnostic woody species such as Elephantorrhizaburkei, Diplorhynchus condylocarpon, Ochna pulchra, Grewia retinervis and Strychnos pungens. Diagnostic grass species include Centropodia glauca, Eragrostis pallens, Schizachyrium jeffreysii, Eragrostis gummiflua, Aristida canescens subsp. ramosa, Aristida diffusa subsp. burkei and Loudetia flavida. The herbaceous layer is sparse and species poor and contains some weak indicator species such as Selaginella dregei, Cineraria parvifolia and Tephrosia longipes.

Prominent woody species include Pseudolachnostylis maprouneifolia, Burkea africana (Species Group O), Strychnos madagascariensis, Terminalia sericea (Species Group P), Xerophyta retinervis (Species Group R) and Vitex rehmannii (Species Group U). Locally dominant grass species include Centropodia glauca, Eragrostis pallens, Schizachyrium jeffreysii, Eragrostis gummiflua, Aristida canescens subsp. ramose, Aristida diffusa, Loudetia flavida (Species Group M), Stipagrostis uniplumis var. uniplumis, Enneapogon cenchroides, Schmidtia pappophoroides, Aristida stipitata subsp. graciliflora (Species Group P) and Loudetia simplex (Species Group R). Some of the locally prominent herbaceous species include Selaginella dregei, Portulaca kermesina, Tephrosia longipes, Indigofera cryptantha var. cryptantha, Orthosiphon labiatus, Isoglossa hypoestiflora, Anacampseros subnuda subsp. subnuda (Species Group M), Waltheria indica (Species Group P), Corchorus kirkii, Commelina africana subsp. lancispatha, Myrothamnus flabellifolius (Species Group R) and Pellaea calomelanos var. calomelanos (Species Group T). 
Acocks (1953) described the various plant communities of the Diplorhynchus condylocarpon-Burkea africana Soutpansberg Leached Sandveld Vegetation as variations of the Mixed Terminalia-Dichapetalum Veld (18b) of the Mixed Bushveld (18). The Burkea africana-Setaria lindenbergiana major community described by Van Staden (2002) and Van Staden and Bredenkamp (2005), the Barleria bremekampii-Diplorhynchus Tree Savanna (Coetzee et al. 1976), as well as the Burkea africanaSetaria sphacelata Undulating Plains, Foot slopes, Terraces and Plateaus Community and the Terminalia sericea-Eragrostis pallens Deep Sandy Lowlands Community described by Henning (2002) from the Waterberg area share many diagnostic species with the Diplorhynchus condylocarpon-Burkea africana Soutpansberg Leached Sandveld major vegetation type of the SC. The coarse vegetation described by Van den Berg (1993) of the Sour and Mixed Bushveld emphasises the heterogeneity of these Veld Types. The Soutpansberg Leached Sandveld MVT shares numerous diagnostic species with the Terminalio sericeae-Combretetea apiculati described by Winterbach (1998) and Winterbach et al. (2000). More specifically, this major vegetation type shares numerous diagnostic species with the Burkea africana-Perotis patens Woodland Alliance described by Van der Meulen (1979).

Due to the nutrient poor nature of this major vegetation type, grazing and browsing fodder production is generally low. Most of the landscape is homogeneous, resulting in low species richness. However, the more rugged landscapes with their higher diversity of micro-habitats are relatively rich in species.

7. Rhus rigida var. rigida-Rhus magalismontanum subsp. coddii Soutpansberg Cool Mistbelt Major Vegetation Type

The Rhus rigida var. rigida-Rhus magalismontanum subsp. coddii Mistbelt vegetation of the SC is situated higher than $1200 \mathrm{~m}$ above sea level and is confined to the mistbelt region. It is associated with Glenrosa and Mispah soil forms (MacVicar et al. 1991) derived from sandstone, quartzite and conglomerate associated with the Fa and Ib Land Types of the Wyllies Poort Geological Formation (Botha 2004b; Patterson \& Ross 2004b). The Champagne soil form (MacVicar et al. 1991) was recorded along some of the localized high-lying wetlands and peatlands.

This MVT is associated with the rugged landscapes of the upper plateaus and crests of the SC. The soils derived from the underlying sandstone and quartzite can be described as extremely shallow, coarse sands. The depth of the soil and the extent of rock cover determine the vegetation structure and species composition within this MVT. During the rainfall season these plant communities are covered in mist on an almost daily basis, contributing towards the abundance of rock and bark lichens and bryophytes. The combination of frequent orographic rain and mist during the summer months leaves the available pockets of soil among the rock sheets drenched and sometimes flooded for extended periods. The deeper soil pockets and the half-weathered matrix of saprolite within the mistbelt can be regarded as the sponge areas, which slowly release water to feed mountain streams over extended periods. During the dry season the shallow sandy soils rapidly dry out. The local and surrounding topography of the landscape plays a major role in the rate of desiccation or water retention of a specific patch of soil. Extended flooding is often a product of water seepage into an area from the surrounding catchments and sponge areas. In cases where water floods the soil for most of the year, wetlands and peatlands have formed. However, these high-lying wetlands and their surrounding catchments are prone to severe periodic droughts. These fluctuating moisture conditions favour those plant species with strategies such as the ephemerals, xerophytes and succulents.

Structurally, the plant communities of the Mistbelt MVT are extremely diverse. It includes peatlands, low open grasslands and islands of short thickets or bush clumps (Edwards 1983). Due to this structural diversity within this major vegetation type, diagnostic species comprise of a mixture of inconspicuous perennials, and do not include any dominant or abundant species. Diagnostic species for this group are presented in species group $\mathrm{Q}$ (Table 1). The most important diagnostic woody species include Rhus rigida var. rigida, Helichrysum kraussii, Cryptolepis cryptolepioides and Parinari capensis subsp. capensis. Other, less constant woody indicator species of this MVT include Olea capensis subsp. enervis, Syzygium legatii, Aloe arborescens, Rotheca myricoides, Euclea linearis, Rhus tumulicola var. meeuseana, Olinia rochetiana, Combretum moggii, Vangueria soutpansbergensis, Coptosperma supra-axillare, Protea caffra subsp. caffra, Elephantorrhiza elephantina, Tetradenia riparia, Apodytes dimidiata subsp. dimidiata, Protea roupelliae subsp. roupelliae, Lopholaena coriifolia, Coptosperma rhodesiacum, Ekebergia pterophylla and Myrsine africana. Diagnostic grass species include Melinis nerviglumis, Brachiaria serrata, Setaria sphacelata var. torta, Trachypogon spicatus and Elionurus muticus. Diagnostic herbaceous species recorded are Fadogia homblei, Coleochloa setifera, Rhynchosia monophylla, Senecio barbertonicus, Crassula swaziensis, Khadia borealis, Vernonia natalensis, Dicoma anomala, Plectranthus neochilus, Gnidia cuneata, Eulophia ensata, Pteridium aquilinum, Kalanchoe sexangularis, Aristea woodii, Anthospermum welwitschii, Vernonia oligocephala, Pentanisia prunelloides subsp. prunelloides, Senecio oxyriifolius, Ipomoea oblongata, Helichrysum cerastioides, Bulbostylis contexta, Hypoxis hemerocallidea and Asparagus falcatus.

Prominent species within this collection of diverse communities include the woody species Rhus rigida var. rigida, Helichrysum kraussii, Cryptolepis cryptolepioides, Parinari capensis subsp. capensis (Species Group Q), Combretum molle, Englerophytum magalismontanum, Rhus magalismontanum subsp. coddii, Mimusops zeyheri (Species Group U) and Maytenus undata (Species Group X). Locally prominent grass species include Melinis nerviglumis, Brachiaria serrata, Setaria sphacelata var. torta, Trachypogon spicatus, Elionurus muticus (Species Group Q) and Loudetia simplex (Species Group R). Due to the topographic heterogeneity of this MVT, and the diversity of plant communities it contains, prominent herbaceous species are only

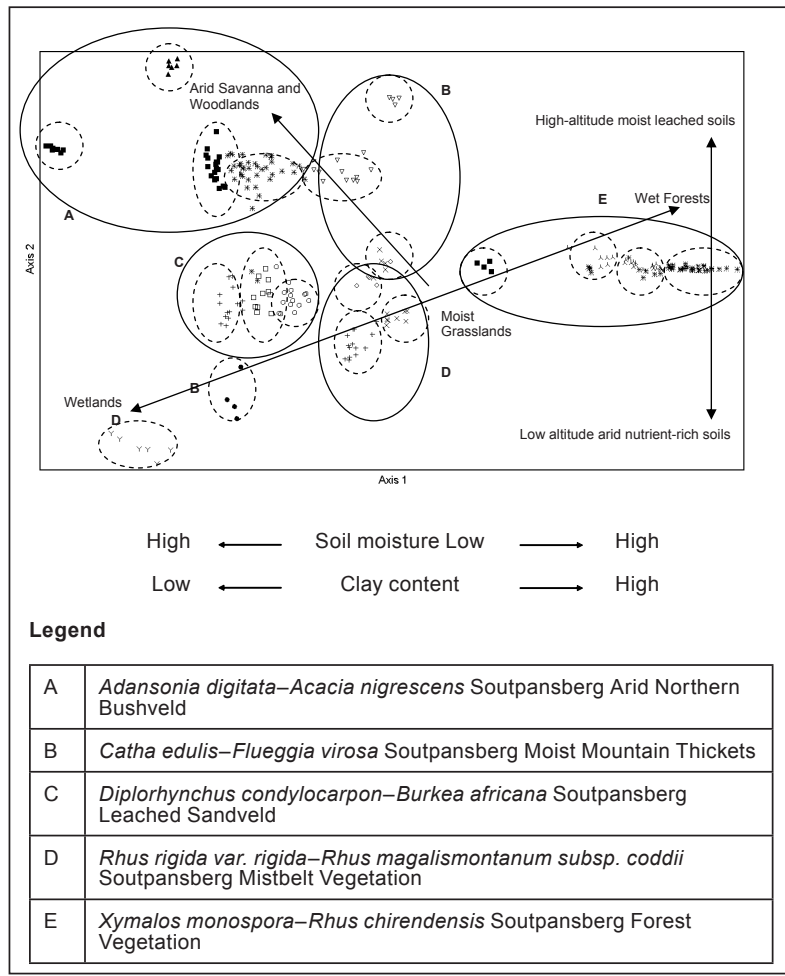

FIGURE 5

Relative positions of relevés along the first and second axis of the DECORANA ordination (Hill 1979b) of the vegetation data from the Soutpansberg Conservancy. 
locally prominent and may include Fadogia homblei, Coleochloa setifera, Rhynchosia monophylla, Senecio barbertonicus, Crassula swaziensis, Khadia borealis, Vernonia natalensis, Dicoma anomala, Plectranthus neochilus, Gnidia cuneata, Wahlenbergia undulata Hypoxis argentea var. argentea, Pteridium aquilinum, Kalanchoe sexangularis, Vernonia oligocephala, Pentanisia prunelloides subsp. prunelloides, Hypoxis hemerocallidea (Species Group Q) and Pellaea calomelanos var. calomelanos (Species Group T).

The Rhus rigida var. rigida-Rhus magalismontanum subsp. coddii Mistbelt vegetation type of the SC is a mosaic of closed bush clumps from the Englerophyto magalismontani-Acacietea caffrae in the savannas (Winterbach et al. 2000), the patches of high-lying, low open grasslands from the Loudetio simplicis-Alloteropsidetea semialatae in the grasslands (Matthews et al. 1994), the Loudetia simplex-Aristida aequiglumis Woodlands, Shrublands and Grasslands (Coetzee 1975) and the Protea caffra-Loudetia simplex Major Community (Van Staden 2002). The bush clump communities show strong floristic affinities with the Rhus tumulicola-Aloe arborescens mist belt bush clumps (Matthews 1991; Matthews et al. 1991) of the north-eastern Mountain Sourveld (Acocks 1953) and the Landolphia capensis-Crassula argyrophylla savanna (Bredenkamp 1975).

Although thegrassland patchessharestructuralsimilarities with the Diheteropogono amplectentis-Proteetum gaguedi described by Matthews et al. (1994) and the Protea caffra-Helichrysum setosum savanna described by Bredenkamp (1975), they show stronger floristic affinities with the grassland patches of the structurally different Helichrysum kraussii-Englerophytum magalismontanum bush clump communities described by Matthews (Matthews 1991; Matthews et al. 1991)

\section{Xymalos monospora-Rhus chirendensis Soutpansberg Forest} Major Vegetation Type

The forests of the SC are confined to the southern slopes of the southern most ridges of the mountain. It is associated with the Glenrosa, Mispah and Shortlands soil forms (MacVicar et al. 1991) derived from basalt, tuff, sandstone, and conglomerate associated with the Fa Land Type of the Sibasa Geological Formation (Botha 2004b; Patterson \& Ross 2004b). The soils are generally rich in organic matter and contain relatively high percentages of clay in areas where basalt and tuff form the underlying geological material. Defining the geology and soil formations is often difficult along this rupture section of the mountain where the upper sedimentary plates have torn and became mixed with volcanic material (Barker 1979, 1983; Bumby 2000). Should vegetation be removed, soil erosion becomes a major problem along the steep southern slopes with its relatively shallow soils and high rainfall.

This major vegetation type is dependent on the orographic rain driven onto the southern slopes by a south-easterly wind during summer. The evergreen high forests are confined to the mistbelt of the mountain, which reaches down as far as $1380 \mathrm{~m}$ above sea level (Geldenhuys \& Murray 1993). Deciduous shrub forest forms a fire resistant ecotone of thickets, which extends to below the mistbelt zone of the southern slopes.

The diagnostic species for this major vegetation type are presented in Species Group V (Table 1). The diagnostic woody species include Xymalos monospora, Zanthoxylum davyi, Celtis africana, Nuxia floribunda, Rhoicissus tomentosa, Kiggelaria africana, Vepris lanceolata, Rapanea melanophloeos, Rothmannia capensis, Brachylaena discolor, Ficus craterostoma, Combretum kraussii, Trichilia dregeana, Trimeria grandifolia, Drypetes gerrardii and Oxyanthus speciosus subsp. gerrardii.

Other prominent woody species include Diospyros whyteana (Species Group W), Maytenus undata, Rhus chirindensis, Cussonia spicata (Species Group X) and Maesa lanceolata (Species Group Y).
A recent classification of forest vegetation data by Von Maltitz et al. (2003) merged the forest communities of the Blouberg, Soutpansberg, North Eastern Escarpment, Mariepskop and Barberton regions under the name Northern Mistbelt Forest. These Afrotemperate forests have been described by numerous authors, and undervarious differentnames, such as Afromontane Forest (Cooper 1985, White 1978), Temperate, Transitional and Scrub Veld Types (Acocks 1953), Uplands Vegetation (Edwards 1967; Moll 1976), Interior Forests (MacDevette et al. 1989), Montane Podocarpus Forest (Cooper 1985; Edwards 1967; Moll 1976), Highland Sourveld (Acocks 1953), Mist Belt Mixed Podocarpus Forest (Cooper 1985; Edwards 1967; Moll 1976) and Natal Mist Belt 'Ngongoni Veld (45) (Acocks 1953).

The evergreen Xymalos monospora-Rhus chirendensis Soutpansberg Forest Vegetation, which forms part of the Northern Mistbelt Forests, share some floristic affinities with the Highland Sourveld, the Dohne Sourveld (44b) and the Natal Mist Belt 'Ngongoni Veld (45), as well as the Coastal Tropical forest patches associated with the 'Ngongoni Veld (5) (Acocks 1953). Important shared species include the valuable timber species Podocarpus falcatus, Podocarpus latifolius and Ocotea kenyensis. These species have been exploited for financial gain to such an extent that they are no longer dominant indicator species within all the Soutpansberg forest associations. They now only occur in relatively high abundances where difficult terrain has inhibited people from accessing these species. This phenomenon of low cover-abundance values of the two Podocarpus species within the SC is therefore an artefact of human intervention (McCracken 1986; Obiri et al. 2002). The historical prominence of the two Podocarpus species therefore justifies the Soutpansberg forests' inclusion into the temperate forest category (Acocks 1953). The Widdringtonia nodiflora dominated cliff forests along the scarp faces of the southern slopes of the Soutpansberg share some weak floristic and structural affinities with the Highland Sourveld (Acocks 1953) and the Widdringtonia nodiflora-Podocarpus latifolius Short Forest of the Waterberg (Van Staden \& Bredenkamp 2006).

\section{Ordination}

The rich diversity of plant communities within the SC and BNR is closely associated with the topographic and geologic diversity of these areas. The variation in topography and geology of the landscape contributes to variation in localised climatic conditions, seasonal precipitation, predictability of precipitation, processes and tempos of weathering, degrees of nutrient leaching from the soil, accumulation tempo of organic matter in the soil and plant available moisture in the soil. An ordination of the vegetation data on these two areas revealed distinct groupings of relevés, which in turn represent different major vegetation types and plant communities. In order to display and interpret these vegetation groupings in an ecologically meaningful manner, it was decided to separate the two distinct clusters representing the SC and the BNR.

\section{Vegetation of the Blouberg Nature Reserve}

A scatter diagram (Fig. 4) displays the distribution of relevés along the second and third ordination axes. The vegetation units are represented as groups and their distribution on the scatter diagram corresponds with certain physical environmental conditions. The lack of any distinct groupings, with some relative distance between groupings, reflects the floristic similarities among the plant communities of the BNR. However, numerous environmental gradients could be correlated with the distribution of the plant communities and individual relevés along the $\mathrm{x}$ - and $\mathrm{y}$-axes.

The second axis (eigen value $=0.5828$ ), orientated along the $\mathrm{x}$-axis, represents gradients in soil depth, soil moisture, surface rock cover, altitude, slope, plant-available moisture and soil texture. Those plant communities along the left side of the diagram represent the dry, flat areas with relatively deep fine- 
grained soils. These include the Eragrostis lehmanniana var. lehmanniana-Sclerocarya birrea subsp. caffra Blouberg Northern Plains Bushveld and the Euclea divinorum-Acacia tortilis Blouberg Southern Plains Bushveld. Those plant communities along the right side of the diagram represent the wetter steep, rocky slopes with relatively shallow coarse sandy soils. These are mostly represented by the Englerophytum magalismontanumCombretum molle Blouberg Mountain Bushveld.

The third axis (eigen value $=0.3918$ ), orientated along the $y$-axis, represents gradients in plant-available moisture, soil depth and slope. Those plant communities along the top of the diagram represent the wetter, steep rocky slopes with relatively shallow coarse sandy soils, represented by the Englerophytum magalismontanum-Combretum molle Blouberg Mountain Bushveld. Those plant communities along the bottom of the diagram represent the dry flat areas with relatively deep finegrained soils, represented by the Eragrostis lehmanniana var. lehmanniana-Sclerocarya birrea subsp. caffra Blouberg Northern Plains Bushveld and the Euclea divinorum-Acacia tortilis Blouberg Southern Plains Bushveld.

\section{Vegetation of the Soutpansberg Conservancy}

The scatter diagram (Fig. 5) displays the distribution of relevés along the first and second ordination axes. The vegetation units are represented as groups and their distribution on the scatter diagram corresponds with certain physical environmental conditions. The very distinct groupings of relevés within the virtual space of the scatter plot are a good indication of the unique nature of each of the different plant communities it represents. These pronounced ordination clusters reinforce those groupings created by the numerical classification done on the available vegetation data of theSC. Numerous environmental gradients could be correlated with the distribution of the plant communities and individual relevés along the $\mathrm{x}$ - and $\mathrm{y}$ - axes.

The first axis (eigen value $=0.9501$ ), orientated along the $x$-axis, represents gradients in plant-available soil moisture and soil clay content. Those plant communities along the left side of the diagram represent the arid areas with relatively sandy soils. These include the Adansonia digitata-Acacia nigrescens Soutpansberg Arid Northern Bushveld and Diplorhynchus condylocarpon-Burkea africana Soutpansberg Leached Sandveld. Those plant communities along the right side of the diagram represent the relatively moist areas with soils that have a higher clay content. These include the Catha edulis-Flueggia virosa Soutpansberg Moist Mountain Thickets and the Xymalos monospora-Rhus chirendensis Soutpansberg Forest Vegetation.

The second axis (eigen value $=0.6412$ ), orientated along the y-axis, represents gradients in plant-available moisture, soil mineral content and altitude. Those plant communities along the top of the diagram represent the lower lying mineral rich arid areas of the SC. These include the Adansonia digitata-Acacia nigrescens Soutpansberg Arid Northern Bushveld and Catha edulis-Flueggia virosa Soutpansberg Moist Mountain Thickets. Those plant communities along the bottom of the diagram represent the moist high altitude major vegetation types on the leached soils of the SC. This includes the. Rhus rigida var. rigidaRhus magalismontanum subsp. coddii Soutpansberg Mistbelt Vegetation.

\section{DISCUSSION}

The results of both the ordination and the classification indicate a considerable difference between the vegetation types of the Blouberg Nature Reserve and the Soutpansberg Conservancy (Fig. 4 \& Fig. 5; Table 1). Species Group F is restricted to the BNR, showing the relationship among the BNR plant communities, and indicates that it has unique vegetation. Although individual major vegetation types of the SC are exceptionally unique (with many diagnostic species, e.g. Species Groups G, I, M, Q and V), no species groups emphasise the coherence of the vegetation of the SC. On the contrary, Species Groups H, K, L, O, P, U and Y indicate the relationship of SC plant communities with some BNR communities.

All these heterogeneous vegetation types and plant communities fall within an estimated area of only 108000 hectares $(1080 \mathrm{~km} 2)$. The conservation of such a biological hotspot should be a provincial, national and international priority (UNESCO 2002; Walker 1989; Wessels et al. 2003). The Soutpansberg Conservancy and the Blouberg Nature Reserve represent numerous major vegetation types of the SCBD, and form the core of present conservation efforts. These nature reserves should act as benchmark sites for the monitoring of the impact of development on the surrounding unprotected areas.

The observed diversity in vegetation patterns is the result of the region's high spatial variation (topography, geology, pedology, extreme localised climate patterns) as well as the region's high temporal variability (irregular climatic cycles, periodic stochastic events) (Gibson et al. 2004). In addition to the environmental factors influencing the observed vegetation and floristic patterns, certain regions of the study area have been altered through intense anthropogenic activities over extended periods of time. The stochasticity with which humans have impacted on this environment has led to even higher levels of spatial and temporal variation in habitat heterogeneity. Numerous stone tools and artefacts indicate that humans have occupied the region on a periodic basis since the Early Stone Age (Coles \& Higgs 1975). More recent times have seen cultures and civilisations such as the Khoisan (Eloff 1979), the people of Mapungubwe (Huffman 1996), the Vhenda people (Nemudzivhadi 1985), and European settlers utilising the region for hunting, livestock farming and the cultivation of crops (Voigt \& Plug 1984).

It is important to identify and to understand the major ecological processes driving this particular ecosystem in order to conserve and manage it effectively (Wessels et al. 2003). The major vegetation patterns seen among the plant communities of the SC and BNR are largely related to the availability of soil moisture and the rate of environmental desiccation (Bond et al. 2003). The underlying geology and soils as well as altitude seem to play only secondary roles in the community composition of these event-driven systems (Krebs 2001). Soil moisture availability within the study area is governed by mainly four environmental factors: (1) the amount of precipitation of atmospheric moisture, (2) the rate of water loss through evaporation (3) the soil's ability to capture and keep moisture within reachable depth of the plant roots, and (4) the available soil water capacity (Kramer 1969; Scott \& Le Maitre 1998). White (1995) defines the available soil water capacity as the amount of water in a soil that is available for plant growth. The upper limit is set by the soil's field capacity (water-saturated soil), while the lower limit is set by the volumetric water content value at which plants lose turgor and wilt, or the permanent wilting point.

The SC and BNR are surrounded by many poverty stricken informal settlements of rural Venda. These people rely on the savanna and forest plant communities to supply grazing, fuel wood, timber and agricultural produce. Approximately $58 \%$ of the province's land area is used for grazing, and $22 \%$ used for agriculture (Hoffman \& Ashwell 2000). The Limpopo Province contributes considerably to the formal economy through its ecotourism, livestock, mining, timber and export fruit and vegetable industries (Adams et al. 2000). Sadly though, this culturally, historically and naturally rich and diverse province of South Africa is a poverty stricken region (Shackleton \& Shackleton 2000). The province's rural communities are often 
locked in a struggle for survival against the frequent and severe droughts. This has led to a culture where "if it does not pay, it does not stay" (Goudie 2000). In addition, the ever-expanding population of South Africa is making increasing demands on the natural resources of the Limpopo region. This will inevitably lead to the expansion of agriculture and industry into marginal and ecologically sensitive areas. In order for the government to plan development, management and conservation sensibly, we need the necessary ecological knowledge of the area. Without this baseline information and insight on the region's driving ecological processes and patterns, the much needed development of the Limpopo's infrastructure and the utilisation of its natural resources will be unsustainable, with only limited short-term benefits for a few selected individuals.

\section{ACKNOWLEDGEMENTS}

We thank the referees for their constructive comments on the manuscript, the National Research Foundation for funding this project and all the landowners within the SC and the management of the BNR for access to gather field data. Thank you to Goro Lodge, Lajuma Mountain Retreat, Medike Mountain Reserve, and the Soutpansberg Herbarium for accommodation during fieldwork.

\section{REFERENCES}

Acocks, J.P.H. 1953. Veld types of South Africa. Memoirs of the Botanical Survey of South Africa, 28: 1-192. ( $3^{\text {rd }}$ edition with updated names and illustrations published in 1988 as Memoirs of the Botanical Survey of South Africa 57: 1-146).

Adams, M., Cousins, B. \& Manona, S. 2000. Land tenure and economic development in rural South Africa constraints and opportunities. In: Cousins, B. (ed.) At the crossroads: Land and Agrarian Reform in South Africa into the 21st Century, Cape Town: University of the Western Cape, pp. 111-128.

Anderson, J.M. (ed.). 2001. Towards Gondwana Alive, Vol. 1 (Assoc. eds. Berger, L., De Wit, M., Fatti, L.P., Holm, E., Rubidge, B. Smith, G., Thackeray, F., \& Van Wyk, B.). Pretoria: Gondwana Alive Society.

Barker, O.B. 1979. A contribution to the geology of the Soutpansberg Group, Waterberg Supergroup, Northern Transvaal. MSc thesis, Johannesburg: University of the Witwatersrand.

Barker, O.B. 1983. A proposed geotectonic model for the Soutpansberg Group within the Limpopo Mobile Belt, Southern Africa. In: Van Biljon, W.J. and J.H. Legg (eds.). The Limpopo Belt. Geological Society of South Africa. Volume 8: 181-190.

Berger, K., Crafford, E., Gaiger, I., Gaiger, M.J., Hahn, N., \& Macdonald, I. 2003. A first synthesis of the environmental, biological and cultural assets of the Soutpansberg. Makhado: Leach Printers \& Signs.

Bezuidenhout, H., Bredenkamp, G.J. \& Theron, G.K. 1994. Phytosociological classes of the western Transvaal grassland, South Africa. Koedoe, 37(1): 1-18.

Bond, W.J., Midgley, G.F. \& Woodward, F.I. 2003. What controls South African vegetation - climate or fire? South African Journal of Botany, 69(1): 79-91.

Botha, M.J. 2004a. Soil mineralogy. In: MacVicar, C.N. (ed.) Land types of the map: 2228 Alldays. Memoirs on the Agricultural Natural Resources of South Africa. Department of Agriculture and Water Supply. Available at: www.arc agric. za/institutes/iscw/main/maps/htm [Accessed 2004]

Botha, M.J. 2004b. Soil mineralogy. In: MacVicar, C.N. (ed.) Land types of the map 2328: Pietersburg. Memoirs on the Agricultural Natural Resources of South Africa. Department of Agriculture and Water Supply. Available: www.arc.agric. $\mathrm{za} /$ institutes/iscw/main/maps/htm [Accessed 2004].

Branch, W.R. 1988. South African Red Data Book - Reptiles and amphibians. South African National Scientific Programmes Report 151. Pretoria: CSIR.
Brandl, G. 2002. The geology of the Alldays area. Explanation, Sheet 2228: Alldays. Council for Geoscience, South Africa, Pretoria.

Bredenkamp, G.J. 1975. 'n Plantsosiologiese studie van die Suikerbosrand-natuurreservaat. MSc thesis, Pretoria: University of Pretoria.

Bredenkamp, G.J., Joubert, A.F. \& Bezuidenhout, H. 1989. A reconnaissance survey of the vegetation of the plains in the Potchefstroom-Fochville-Parys area. South African Journal of Botany 55(2): 199-206.

Bredenkamp, G.J. \& Van Rooyen, N. 1996. 34. Rocky Highveld Grassland. In: Low, A.B. and A.G. Rebelo (eds.). Vegetation of South Africa, Lesotho and Swaziland.p 39, Pretoria: Department of Environmental Affairs and Tourism.

Brown, L.R. 1997. A plant ecological study and wildlife management plan of the Borakalalo Nature Reserve, North West Province. PhD thesis. Pretoria: University of Pretoria.

Bumby, A.J. 2000. The geology of the Blouberg Formation, Waterberg and Soutpansberg Groups in the area of Blouberg Mountain, Northern Province, South Africa. PhD thesis. Pretoria: University of Pretoria.

Coetzee, B.J. 1975. A phytosociological classification of the Rustenburg Nature Reserve. Bothalia, 11: 561-580.

Coetzee, B.J., Van Der Meulen, F., Zwanziger, S., Gonsalves, P. \& Weisser, P.J. 1976. A phytosociological classification of the Nylsvlei Nature Reserve. Bothalia 12: 137-160.

Coles, J.M. \& Higgs, E.S. 1975. The archaeology of early man. Great Britain: Peregrine Books.

Cooper, K.H. 1985. The conservation status of indigenous forests in Transvaal, Natal and O.F.S., South Africa. Durban: Wildlife Society of South Africa.

Du Preez, P.J. \& Bredenkamp, G.J. 1991. Vegetation classes of the southern and eastern Orange Free State (Republic of South Africa) and the highlands of Lesotho. Bloemfontein: Navorsinge van die Nasionale Museum, Volume 7, pp. 477-526.

Edwards, D. 1967. A plant ecological survey of the Tugela River Basin. Botanical Survey of South Africa Memoir No. 36. Pietermaritzburg: Town and Regional Planning Commission.

Edwards, D. 1983. A broad-scale structural classification of vegetation for practical purposes. Bothalia, 14: 705-712.

Eloff, J.F. 1979. III Die kulture van Greefswald - 'n argeologiese studie van die Ystertydperkkulture op die plaas Greefswald. Pretoria: University of Pretoria.

Foord, S.H., Dippenaar-Schoeman, A.S. \& Van Der Merwe, M 2002. A checklist of the spider fauna of the Western Soutpansberg, South Africa (Arachnida: Araneae). Koedoe, 45(1): 35-43.

Foord, S.H. \& Dippenaar-Schoeman, A.S. 2003. Spiders (Araneae). In: Berger, K., Craffort, J.E., Graiger, I., Graiger, M.J., Hahn, N. \& Macdonald, I. (eds.). A first synthesis of the environmental, biological and cultural assets of the Soutpansberg. Makhadu: Leach Printers \& Signs, pp. 45-50.

Fuls, E.R., Bredenkamp, G.J., Van Rooyen, N. \& Theron, G.K. 1993. The physical environment and major plant communities of the Heilbron-Lindley-Warden-Villiers area, northern Orange Free State. South African Journal of Botany, 59(4): 345-359.

Geldenhuys, C.J. \& Murray, B. 1993. Floristic and structural composition of Hanglip forest in the Soutpansberg, Northern Transvaal. South African Forestry Journal, 165: 9-20.

Germishuizen, G. \& Meyer, N.L. 2003. Plants of Southern Africa: an annotated checklist. Strelitzia, 14: 1-1231.

Gertenbach, W.P.D. 1983. Landscapes of the Kruger National Park. Koedoe, 26: 9-121.

Gibson, L.A., Wilson, B.A. \& Aberton, J.G. 2004. Landscape characteristics associated with species richness and occurrence of small native mammals inhabiting a coastal heathland: a spatial modelling approach. Biological Conservation, 120(1): 75-89.

Goudie, A. 2000. The human impact on the natural environment. Oxford: Blackwell Publishers. 
Hahn, N. 1994. Tree list of the Soutpansberg. Pretoria: Fantique Publishers.

Hahn, N. 1996. Rubiaceae. A new species of Vangueria from the Soutpansberg. Bothalia, 27(1): 45-48.

Hahn, N. 1997. Rare and endangered flora of Tshikondeni. Inhouse manuscript of the Tshikondeni Mining Company (Pty) Ltd. Tshikondeni.

Hahn, N. 1999. Rubiaceae. A new species of Pavetta from the Soutpansberg, South Africa. Bothalia, 29(1): 107-109.

Hahn, N. 2002. Endemic flora of the Soutpansberg. MSc thesis, Pietermaritzburg: University of Natal.

Hahn, N. 2003. Endemic flora. In: Berger, K., Crafford, E., Gaiger, I., Gaiger, M.J., Hahn, N. \& Macdonald, I. (eds.). A first synthesis of the environmental, biological and cultural assets of the Soutpansberg. Makhado, Leach Printers \& Signs.

Hahn, N. Endemism and biogeography. PhD thesis, in preparation. Pretoria: University of Pretoria.

Harrison, J.A., Allan, D.G., Underhill, L.G., Herremans, M., Tree, A.J., Parker, V. \& Brown, C.J. 1997. The atlas of southern African birds. Avian Demography Unit, Bird Life South Africa.

Hennekens, S.M. \& Schamineé, J.H.J. 2001. Turboveg, a comprehensive database management system for vegetation data. Journal of Vegetation Science, 12: 589-591.

Henning, B.J. 2002 The relevance of ecosystems to ecotourism in the Waterberg Biosphere Reserve. PhD thesis, Pretoria: University of Pretoria.

Hill, M.O. 1979a. TWINSPAN - a FORTRAN program for arranging multivariate data in an ordered two-way table by classification of individuals and attributes. New York: Cornell University.

Hill, M.O. 1979b. DECORANA - a FORTRAN program for detrended correspondence analysis and reciprocal averaging. New York: Cornell University.

Hockey, P.A.R., Dean, W.R.J. \& Ryan, P.G. 2005. Roberts - Birds of Southern Africa ( $7^{\text {th }}$ edition). The Trustees of the John Voelcker Bird Book Fund, Cape Town.

Hoffman, T. \& Ashwell, A. 2000. Land degradation: Northern Province fact sheet. Cape Town, Salty Print.

Huffman, T. N. 1996. Archaeological evidence for climatic change during the last 2000 years in southern Africa. Quaternary International, 33: 55-60.

Kent, M. \& Coker, P. 1996. Vegetation description and analysis: a practical approach. John Wiley \& Sons Ltd., Sussex, England.

Kramer, P.J. 1969. Plant and soil water relationships: a modern synthesis. New Delhi, United States of America, McGrawHill Publishing Company.

Krebs, C.J. 2001. Ecology: the experimental analysis of distribution and abundance $\left(5^{\text {th }}\right.$ edition). San Francisco: Benjamin Cummings.

Louw, A.J. 1970. Mopane veld noord van die Soutpansberg. DSc thesis, Pretoria: University of Pretoria.

Lubke, R.A. \& Mackenzie, B. 1996. Afromontane Forest. In: Low, A.B. and A.G. Rebelo (eds.). Vegetation of South Africa, Lesotho and Swaziland. Pretoria: Department of Environmental Affairs and Tourism, p. 12

MacDevette, D. R. 1993. The woody vegetation of the Zululand coastal dunes. In: Van Der Sejde (ed.). South African forestry handbook/Suid-Afrikaanse bosbouhandboek. Southern African Institute of Forestry, Pretoria, pp. 633-637.

MacDevette, D.R., Mac Devette, D.K., Gordon, I.G. \& Bartholomew, R.I.C. 1989. Floristics of the Natal indigenus forests of southern Africa. Occasional Report 45. Pretoria: Foundation for Research and Development.

MacVicar, C.N., Bennie, A.T.P. \& De Villiers, J.M. 1991. Soil classification: A taxonomic system for South Africa. Pretoria: Department of Agricultural Development.

Matthews, W.S. 1991. Phytosociology of the North-eastern Mountain Sourveld. MSc thesis, Pretoria: University of Pretoria.

Matthews, W.S., Bredenkamp, G.J. \& Van Rooyen, H. 1991. The grassland-associated vegetation of the Black Reef Quartzite and associated large rocky outcrops in the north-eastern mountain sourveld of the Transvaal Escarpment. South African Journal of Botany, 57: 143-150.

Matthews, W.S., Bredenkamp, G.J. \& Van Rooyen, N. 1994. The phytosociology and syntaxonomy of relatively low-altitude areas in the North-eastern Mountain Sourveld, in the eastern Transvaal escarpment region. Koedoe, 37(2): 73-87.

McCracken, D.P. 1986. The indigenous forests of colonial Natal and Zululand. Natalia, 16: 19-38.

McCune, B. \& Mefford, M.J. 1999. PC-ORD for Windows. Multivariate analysis of ecological data, version 4.10. MjM Software, Gleneden Beach, Oregon, U.S.A.

Moll, E.J. 1976. The vegetation of the Three Rivers region, Natal. Natal Town and Regional Planning Reports 33. Pietermaritzburg: Town and Regional Planning Commission.

Mueller-Dombois, D. \& Ellenberg, H. 1974. Aims and methods of vegetation ecology. New York: Wiley.

Nemudzivhadi, M. 1985. When and what? An introduction to the evolution of the history of Venda. Thohoyandou: Department of Land Affairs.

Obermeyer, A.A., Scweickerdt, H.G. \& Verdoorn, I.C. 1937. An enumeration of the plants collected in the northern Transvaal. Bothalia, 3(2): 223-258.

Obiri, J., Lawes, M. \& Mukolwe, M. 2002. The dynamics and sustainable use of high-value tree species of the coastal Pondoland forests of the Eastern Cape Province, South Africa. Forest Ecology and Management, 199: 131-148.

Olivier, J. \& Rautenbach, C.J. 2002. The implementation of fog water collection systems in South Africa. Atmospheric Research, 64(4): 227-239.

Patterson, D.G. \& Ross, P.G. 2004a. Soil mineralogy. In: MacVicar, C.N. (ed.). Land types of the map 2228 Alldays. Memoirs on the Agricultural Natural Resources of South Africa. Department of Agriculture and Water Supply. Available: www.arc.agric.za/institutes/iscw/main/maps/ htm [Accessed 2004].

Patterson, D.G. \& Ross, P.G. 2004b. Soil mineralogy. In: MacVicar, C.N. (ed.). Land types of the map 2328 Pietersburg. Memoirs on the Agricultural Natural Resources of South Africa. Department of Agriculture and Water Supply. Available at: www.arc.agric.za/institutes/iscw/main $/ \mathrm{maps} / \mathrm{htm}$ [Accessed 2004]

Schutte, J.M. 1971. Die onttrekking van water uit die newellaag en lae wolke by Mariepskop. S.A. Department of Water Affairs, Division Hydrological Research, Technical note 20: 1-21.

Scott, D.F. \& Le Maitre, D.C. 1998. The interaction between vegetation and groundwater: research priorities for South Africa. Pretoria, WRC Report no. 730/1/98. Water Research Commission.

Shackleton, C.M. \& Shackleton, S.E. 2000. Direct use values of secondary resources harvested from communal savannas in the Bushbuckridge Lowveld, South Africa. Journal of Tropical Forest Products 6: 28-47.

Siebert, S.J. 2001. Vegetation on the ultramafic soils of the Sekhukhuneland Centre of Endemism. PhD thesis, Pretoria: University of Pretoria.

Siebert, F., Bredenkamp, G.J. \& Siebert, S.J. 2003. A comparison of Mopane vegetation in South Africa, Namibia and Zimbabwe. Bothalia, 33(1): 121-134.

Smit, J.H.L. 2000. Phytosociology and veld management of the eastern Kalahari Thornveld. MSc thesis, Pretoria: University of Pretoria.

South African Weather Buro 2004. South African Weather Service. Available: http://www.weathersa.co.za [Accessed 2004].

Stirton, C.H. 1982. A new species of Rhynchosia from Venda. Bothalia, 14(1): 76-77.

Unesco. 2002. Convention concerning the protection of the world cultural and natural heritages. UNESCO World Heritage Centre, France http://www.unesco.org [Accessed 2004] 
Van den Berg, H.M. 1993. Die identifisering en kartering van veldekotope in die noordwes-Transvaalse suur-en gemengdebosveld. MSc thesis, Pretoria: University of Pretoria.

Van der Meulen, F. 1979. Plant sociology of the western Transvaal Bushveld, South Africa: a syntaxonomic and synegological study. Dissertationes Botanicae, 49: 1-192.

Van Jaarsveld, E. \& Hardy, D. 1991. The Blouberg botanical expedition, with specific reference to the succulent vegetation. Aloe, 28(3): 78-83.

Van Rooyen, N. \& Bredenkamp, G.J. 1996. 17. Sweet Bushveld.. In: Low, A.B. and A.G. Rebelo (eds.). Vegetation of South Africa, Lesotho and Swaziland. Pretoria: Department of Environmental Affairs and Tourism. p. 21.

Van Staden, P.J. 2002. An ecological study of the plant communities of Marakele National Park. MSc thesis, Pretoria: University of Pretoria.

Van Staden, P.J. \& Bredenkamp, G.J. 2005. Major plant communities of the Marakele National Park. Koedoe, 48(2): $59-70$.

Van Staden, P.J. \& Bredenkamp, G.J. 2006. A floristic analysis of forest and thicket vegetation of the Marakele National Park. Koedoe, 49(1): 15-31.

Van Wyk, A.E. 1984. A new species of Combretum from Venda and taxonomic notes on the section Angustimarginata (Combretaceae). South African Journal of Botany, 3(2): $125-135$

Van Wyk, A.E. \& Smith, G.F. 2001. Regions of floristic endemism in southern Africa. Pretoria: Umdaus Press.

Van Wyk, P. 1994. Field guide to the trees of the Kruger National Park. Cape Town: Struik Publishers.

Van Wyk, A.E. 1996. Biodiversity of the Maputaland Centre. In: Van Der Maesen, L.J.G., X.M. Van Der Burgt and J.M. Van Medenbach De Rooy (eds.). The biodiversity of African plants. Wageningen: Kluwer Academic Press. pp. 198-207.
Voigt, E.A. \& Plug, I. 1984. Happy Rest: the earliest Iron Age fauna from the Soutpansberg. South African Journal of Science, 80: 22-227.

Von Maltitz, G.P., Mucina, L., Geldenhuys, C., Lawes, M., Eeley, H., Adie, H., Vink, D., Flemming, G. \& Voster, T. 2003. Classification system for South African Indigenous Forests: an objective classification for the Department of Water Affairs and Forestry. Environmentek report ENV-P-C 2003-017. Pretoria: CSIR.

Walker, B.1989. Diversity and stability inecosystem conservation. In: Western, D. and M. Pearl (eds.). Conservation for the twenty-first century. New York: Oxford University Press.

Westoby, M., Walker, B. \& Noy-Meir, I. 1989. Opportunistic management for rangelands not at equilibrium. Journal of Rangeland Management, 42(4): 266-274.

Wessels, K.J., Reyers, B., Van Jaarsveld, A.S. \& Rutherford, M.C. 2003. Identification of potential conflict areas between land transformation and biodiversity conservation in north-eastern South Africa. Agriculture, Ecosystems and Environment, 95: 157-178.

White, F. 1978. The Afromontane Region. In: Werger, M.J.A. (ed.). Biogeography and ecology of southern Africa. The Hague, Junk, pp. 463-513.

White, R.E. 1995. Introduction to the principles and practices of soil science ( $2^{\text {nd }}$ edition). Oxford: Blackwell Sciences.

Winterbach, R. 1998. A phytosociological synthesis of Acacia tortilis communities in the north-western savanna of South Africa. MSc thesis, Pretoria: University of Pretoria.

Winterbach, R., Bredenkampl, G.J., Deutschlander, M.S. \& Mucina, L. 2000. Preliminary syntaxonomic scheme of vegetation classes for the Central Bushveld of South Africa. In: Whyte, P.S., Mucina, L., Leps, J.S. \& Van Der Maarel, E. (eds.). Proceedings IAVS Symposium. Upsala: Opulus Press. pp. 123-127. 